\title{
Les migrations africaines dans l'enfance, des parcours individuels entre institutions locales et institutions globales
}

\section{Résumé}

Bien que concernés massivement par la migration, les enfants africains sont encore peu visibles dans la littérature tant grise que scientifique. A partir d'une revue critique de la littérature, cet article explore les raisons de cet état de fait en suivant le fil rouge de la construction historique du paradigme de l'enfant africain. Qu'il s'agisse de la confusion entre enfants et jeunes, de la domination du modèle migratoire masculin de travail ou encore de l'oscillation entre vulnérabilité et agency, la complexité, la diversité et la spécificité des enfants migrants et de leur parcours ne peuvent plus être ignorées. La nécessaire analyse de l'articulation entre parcours individuels et institutions passe obligatoirement par une réflexion sur la notion de « voix » de ces acteurs particuliers que sont les enfants.

Mots clefs : migration, enfants, jeunes, agency, institutions locales et globales, parcours individuels

\section{African Migration in Childhood : Individual trajectories caught between local and global institutions}

\section{Abstract}

While African children are massively involved in migration, they remain occluded in grey as well as scholarly literature. Based on a critical review of the literature, this article explores the reasons for this situation by examining the common themes at the heart of the historical construction of the African child paradigm. Despite the common confusion between "children" and "youth", the domination of the male labor migratory model or the oscillation between vulnerability and agency, the complexity, diversity and specificity of these migrating children and their trajectories cannot be ignored. The articulation between individual trajectories and institutions requires that the notion of "voice" be truly examined in the case of these specific actors.

Keywords : migration, children, youth, agency, local and global institutions, individual trajectories

\footnotetext{
* Anthropologue, Chargée de cours en anthropologie, Institut des Sciences Humaines et Sociales (Université de Liège), Elodie.Razy@ulg.ac.be

Historienne, Lecturer in the History of Africa, History Department, SOAS (University of London), mr28@soas.ac.uk
} 
Dans le monde académique - comme dans les politiques publiques nationales et internationales relayées par les canaux médiatiques -, des figures de l'enfance contrastées tracent une frontière entre l'Euro-Amérique et le reste du monde : si l'enfant-roi, et dans certains cas son corrolaire l'enfant 'rebelle', semblent actuellement dominer les discours 'au Nord', ce sont les images d'enfant-victime (Guillermet, 2010 ; Fassin, 2010), d'enfant exploité (Schlemmer, éd., 1996), ou encore d'enfant source d'espoir pour l'avenir, qui s'imposent le plus souvent lorsqu'il est question des pays 'du Sud' et tout particulièrement de l'Afrique. Ces figures contrastées attestent de l'ambivalence et de l'inquiétude que soulève cette catégorie de la population dont les constructions sont le produit d'époques et de cadres culturels spécifiques (McNee, 2004). Bien que la ligne de démarcation, socialement et culturellement construite entre ces deux entités abstraites que sont le Nord et le Sud, soit peu interrogée de manière critique à propos des enfants, l'examen de certaines figures médiatrices comme celle de l'enfant migrant nous renseigne sur les rapports historiques complexes entre différentes régions du monde, et notamment entre l'Afrique et l'Europe. Profils d'enfants 'à discipliner' ou 'à sauver', droits de l'enfant ou encore droit à l'enfant sont en effet le fruit d'une évolution historique et politique ${ }^{1}$ qui a poussé au premier plan une catégorie d'acteurs jusqu'ici peu prise en compte et ainsi suscité un intérêt grandissant que la recherche n'a cessé depuis de relayer.

${ }^{1}$ Cf. Stephens (éd.) (1995); Scheper-Hughes et Sargent (éds.) (1998); Segalen (2010). Plusieurs des notions et thématiques centrales dans l'appréhension des migrations d'enfants, qui sont abordées de manière transversale dans le numéro, s'inscrivent dans le cadre de la convention des droits de l'enfant, soit parce qu'elles y figurent comme un droit (éducation,

$X$ protection, doit à l'expression, etc.), soit parce qu'elles sont envisagées comme porteuses de risque (le déplacement).

3 Pour une analyse des défis méthodologiques et épistémologiques que posent ces études, voir notamment; Hardman (1993); Weiss (1993); Kubik (1995), ainsi que plusieurs contributions in Renner (éd.) (1995); Gottlieb (2000) ; Danic, Delalande et Rayou (2006); Bluebond-Langner et Korbin (2007); Montgomery (2009). 
L'analyse des expériences migratoires des enfants est au carrefour de nombreuses disciplines instituées (histoire, anthropologie, psychologie, psychiatrie, psychanalyse, sociologie, sciences de l'éducation, etc.), de champs de recherche plus récents (Children Studies) ${ }^{3}$, mais également de différentes sphères institutionnelles (Etats, institutions internationales) et professionnelles (médicale, paramédicale, sociale, $O N G$ ) qui souvent ne dialoguent que peu entre elles. De par leur position liminaire, leur statut de passeurs de frontières, les enfants migrants complexifient l'approche des migrations. Sans doute plus que pour d'autres, leur expérience migratoire ne cadre pas toujours avec les rôles qui leur sont assignés par les adultes. Entrer dans les vies de ces enfants révèle les multiples enjeux dont ils sont l'objet et les vecteurs, et permet de mieux comprendre leurs mondes migratoires.

Nous adoptons ici une définition 'totale' de la migration afin de montrer les interconnexions entre les différentes formes de migration, dont les frontières s'avèrent souvent floues (Cordell, Gregory et Piché, 1996). Pour reprendre la définition de Moch (1987) dans le contexte africain, par migration, nous entendons tout changement de résidence, temporaire ou permanent, qui se fait au-delà de la limite définie par le lieu de vie d'une communauté déterminée, que cela soit un village ou une ville, que ces migrations soient locales, régionales ou intra/intercontinentales, ce qui inclut à la fois les migrations saisonnières et les migrations à plus long terme ${ }^{5}$.

La littérature fait rarement la distinction entre mobilités, migration et circulation. Nous retenons ici le terme migration qui nous apparaît plus large dans son acceptation que celui de mobilité. La notion de mobilité, en ne retenant finalement que le mouvement en lui-même, tend de fait à exclure la possibilité d'immobilité/d'arrêt dans le parcours migratoire. Associée à la notion de mobilité sociale, son utilisation peut également amener à concentrer le questionnement scientifique sur le succès ou l'échec de la migration, son aspect émancipateur ou aliénant, jugements qui s'avèrent finalement très subjectifs et somme toute essentialisants. De même, le terme circulation, qui renvoie à une forme particulière de migration faite de déplacements successifs, d'allers-retours inscrivant les migrants (adultes ou enfants) dans un rapport particulier au temps et à l'espace, ne sera pas employé comme terme générique car il exlut d'office les migrations uniques et définitives.

\footnotetext{
${ }^{5}$ Sur une discussion quant aux différents critères retenus en sciences sociales pour définir la notion de migration, voir Domenach et Piquet (1995).
} 
Si les recherches sur les enfants d'une part, et les recherches sur les migrations en Afrique d'autre part, ont connu un développement certain ces vingt dernières années, il semble qu'aujourd'hui encore, les dynamiques familiales migratoires en Afrique restent sous-étudiées (Razy et BabyCollin, éds., 2011) et que le thème des enfants migrants africains n'ait finalement pas encore suscité tout l'intérêt qu'il mérite.

Des études importantes sur les expériences migratoires des enfants en Afrique ont certes été menées ces dernières années, tant au niveau sociologique, anthropologique (entre autres: Lambert, 2002; Whitehead and Hashim, 2005; Hashim, 2005, 2006 et 2007; Thorsen, 2006, 2007 ; Hertrich et Lesclingand, 2007; Whitehead, Hashim et Iversen, 2007; Collinson, 2008; Hashim et Thorsen, 2011) qu'historique (Beinart, 1991 ; Rich, 2010 ; Lord, 2010 et 2011) $)^{6}$. Si ces travaux permettent notamment de montrer que les enfants sont socialement et historiquement des acteurs à part entière de la migration, qu'ils migrent seuls ou avec des adultes, il est cependant important de remarquer, comme le souligne Lord (2010:33), que dans la plupart de ces travaux, c'est la dimension plus sociale que spatiale de la migration qui est analysée. Or, comme noté précédemment, la focalisation sur la mobilité sociale (notamment, la migration 'émancipatrice', voir infra), présente le risque d'empêcher toute prise en compte de la diversité des situations migratoires.

Mais de quels enfants parle-t-on? Dans la littérature sur l'Afrique, on identifie plusieurs déclinaisons de la figure de l'enfant victime qui ne peuvent être comprises qu'au regard les unes des autres dont celle de l'enfant esclave, de l'enfant malnutri, de l'enfant orphelin, de l'enfant exploité, de l'enfant soldat, de l'enfant des rues ou encore de l'enfant sorcier. L'enfant migrant est de fait rarement présent.

Nous nous interrogerons dans un premier temps sur les différents modèles sociaux et historiques de l'enfance en Afrique et sur la place des différentes figures de l'enfant migrant dans ces modèles. La pertinence d'un modèle migratoire propre à la catégorie des enfants sera ensuite abordée au regard du modèle masculin dominant, de la notion d'agency et de la complexité des situations migratoires dans l'enfance. Enfin, la présentation et l'analyse des articles du numéro permettront notamment de s'interroger sur les problèmes méthodologiques liées à la question de la voix des enfants dans l'expérience migratoire, ce qui nous amènera à montrer que les migrations dans l'enfance se retrouvent au carrefour d'enjeux sociaux,

\footnotetext{
${ }^{6} \mathrm{C}$ 'est dans la littérature grise que l'ont trouve le plus grand nombre de travaux sur les enfants migrants africains et son corrolaire le trafficking, le plus souvent sous la forme de rapports (cf. Child Migration Researcher Network, University of Sussex).
} 
économiques et politiques complexes, entre institutions locales et institutions globales.

\section{ENFANTS, ENFANCE ET JEUNESSE D'AFRIQUE : FIGURES ET MODELES MULTIPLES}

S'il est aujourd'hui avéré que les notions d'enfance et d'enfant sont socialement et culturellement construites, interroger la place des enfants migrants dans les sociétés contemporaines, et plus particulièrement africaines, implique le nécessaire mais néanmoins encore trop rare recours à une perspective historique croisée, laquelle permet notamment de mieux saisir les influences mutuelles et les implications sur la longue durée des contacts entre sociétés européennes et sociétés africaines.

Analyser la succession, la superposition et la rencontre des différents 'modèles d'enfances' (Bonnet, Rollet, de Suremain, éds., sous presse), notamment au XXè siècle, permet ainsi de mieux saisir la manière dont s'est construit le paradigme de l'enfant africain.

Cependant, il demeure extrêmement difficile d'obtenir des données sur les constructions anciennes de l'enfance en Afrique. Pour retracer les expériences de vie des enfants africains sur la longue durée, les chercheurs sont souvent confrontés à un problème de documentation, même si la découverte de nouvelles sources ou la réévaluation de certaines déjà connues permettent de plus en plus d'y remédier (manuscripts arabes, récits des premières missions chrétiennes, des voyageurs, histoire orale, etc.) Malgré ces données parcellaires, on connaît mieux aujourd'hui l'importance des enfants dans l'histoire africaine ${ }^{7}$ : ils furent très nombreux parmi les victimes des traites internes, trans-sahariennes et transatlantiques, ils ont été et demeurent une source de main-d'œuvre cruciale pour l'économie familiale et communautaire (Diptee et Klein, 2010; Klein, 2011; Getz, $2011),{ }^{8}$ ils ont souvent joué un rôle central dans l'introduction de nouvelles pratiques religieuses (De Waal et Argenti, 2002). Ils furent au cœur de nombreux enjeux coloniaux et post-coloniaux de pouvoir, notamment en tant que métisses (White, [1999] 2004 ; Lee, 2009), orphelins (Guillermet, 2010), christianisés (de Benoist, 1987), islamisés (Rodet, 2011), écoliers (Bouche, 1975; Sow, 2009 ; Barthélémy, 2011), malades (Ndao, 2003 ;

\footnotetext{
${ }^{7}$ Grâce notamment à des publications spécifiques sur ce thème dont voici une liste non exhaustive : Diop (éd.) (2003); Grier (2004 et 2005); Diptee et Klein (2010); Campbell, Miers et Miller (éds.) (2009 et 2011); Lord (2010 et 2011).

${ }^{8}$ Sur les enfants comme source de main-d'œuvre et de valeur dans l'histoire africaine, voir notamment : Klein et Roberts (1987); Beinart (1991) ; Schmidt (1992); Falola et Lovejoy, éds. (1994): Guyer et Belinga (1995): Price (1996): Gottlieb (2004); Grier (2004 et 2005); Shadle (2006); Lord (2011).
} 
Dieng, 2003) ${ }^{9}$, apprentis et jeunes travailleurs (Moran, 1992 ; Tamba, 2003 ; Diallo, 2003 ; Kom, 2009 ; Coe, 2010$)^{10}$.

On notait qu'en 2006, la population âgée de moins de 15 ans représentait en Afrique subsaharienne $44 \%$ de la population, ce qui en fait incontestablement la plus jeune région du monde. On continue cependant à ne connaitre que trop peu de choses sur ces enfants, des générations passées aux générations actuelles.

Qui sont-ils? Parle-t-on de filles ou de garçons? D'enfants allaités ou d'enfants sevrés? Enfant, adolescent, jeune recouvrent-ils les mêmes réalités partout, à toutes les époques et dans tous les contextes ? Sont-ils des termes interchangeables? Quel rôle a pu jouer et joue encore le statut social de la famille sur la définition de l'enfance et la place de l'enfant dans la société ?

\section{Définir un enfant...}

Afin de répondre à ces questions, il convient de s'intéresser à une dimension peu abordée dans les travaux cités et pourtant cruciale, celle de la périodicisation du cycle de vie, et pour ce qui nous intéresse plus particulièrement ici, de la période allant des débuts de la vie à l'entrée dans l'âge adulte dont les critères de fixation sont souvent déliés de leur contexte historique, social et culturel. Les passages du statut d'enfant à celui d'adulte ne sont en effet pas uniformes en Afrique ni d'ailleurs au sein d'une même société et à une époque donnée (Guidetti, Lallemand et Morel, éds., 1997). De plus, le regard porté sur ces passages évolue (Montgomery, 2008). Si les trajectoires vers le statut d'adulte peuvent être multiples et varier d'une personne à l'autre (Notermans, 2004) et si les modèles figés ne permettent pas de saisir la spécificité des vies d'enfants, force est de constater que les étapes du cycle de vie partagées au sein d'une société ou d'une communauté continuent de servir de référent, d'idéal ou encore de baromètre à partir desquels se déclinent les situations particulières.

Peu de sociétés africaines utilisent l'âge pour différencier les enfants/cadets des adultes ${ }^{11}$, mais s'appuient sur une combinaison de

\footnotetext{
${ }^{9}$ Notons également la longue liste, mais qui ne peut être détaillée ici faute de place, des études sur les enfants malades du VIH / sida, ou victimes collatérales de la maladie, en Afrique.

${ }^{10}$ Il faudrait également inclure ici la très longue liste des ouvrages sur le travail des enfants en Afrique. Pour un début de liste notamment du point de vue historique, voir note 5.

${ }^{11}$ En Europe au XIXè siècłe, les médecins distinguent les différentes périodes comme suit :

$\chi \quad$ la petite enfance (jusqu'à deux ans et demi), la moyenne enfance (de trois à six-sept ans) généralement à cette époque, on distingue la première enfance (jusqu à sept ans) de la seconde enfance (ou moyenne enfance) jusqu'à la puberté (douze, treize ans pour les filles et
} 
variables telles que le statut social, le niveau de dépendance, le sexe et les relations de parenté (Mcnee, 2004 ; Christiansen, Utas et Vigh, éds, 2006 ; Aguilar, éd., 2007 ; Diptee et Klein, 2009), produisant pour certains auteurs "l'âge social" (Grier, 2004). C'est donc ici plutôt le statut dans la société qui définit l'âge et non l'âge qui définit le statut (Mcnee, 2004). Ce statut dépend également de l'acquisition de compétences psychomotrices et sociales, de la construction de relations (Rabain, [1979]1994; Bonnet, 1988; Bril, 1988; Riesman, 1992; Lancy, 1996; Gottlieb, 2004 ; Razy, 2007a, etc.), notamment durant les premières années, puis de la capacité physique individuelle de travail, du besoin social et économique en force de travail et de la volonté de chacun d'exécuter certaines tâches requises par les personnes ayant le statut d'adulte (Hashim et Thorsen, 2011). Dans ce sens, Honwana (2005) parle par exemple pour l'Angola et le Mozambique en termes de rôles sociaux, d'attentes et de responsabilités tout en précisant bien que l'âge est cependant utilisé lorsque les gens évoquent des limites d'âge qui permettent de situer les différentes étapes perçues.

Dans une certaine mesure, l'enfant du lignage des sociétés paysannes d'Afrique et l'enfant de la maisonnée des sociétés paysannes d'Europe (système de parenté à maison) peuvent être comparés dans leur double rôle de producteur et de reproducteur (main-d'œuvre et maillon de la tranmission) (Segalen, 2010). Mais à partir du XIXè siècle, la transposition du projet colonial en Afrique uniformise une diversité des sociétés alors peu (re)connue tout en 'redistribuant' les cartes des différences ethniques : qu'il s'agisse de la partition entre sociétés à classes d'âge et sociétés sans classes d'âge, des diverses déclinaisons des stratifications sociales et des systèmes de parenté, de la présence d'initiations, de rites de passage institués marquant l'acquisition de l'identité sexuelle, d'un statut rompant avec l'enfance sans pour autant signifier l'entrée dans l'âge adulte (souvent associée au mariage, pouvant lui-même être lié à la puberté, voire à la naissance du premier enfant), ou encore de simples passages marqués, comme le sevrage, sans pour autant être ritualisés collectivement ${ }^{12}$. Autant de traits qui pointent les difficultés à appréhender le cycle de vie en termes d'âges universels et fixes et l'impossibilité de faire abstraction des

quatorze ans pour les garçons) (Rollet, 2001). La fin de l'enfance se situe donc à la puberté, mais on note la différence marquée entre les sexes. Ce n'est qu'à la fin du XIXè siècle que 'l'adolescence' est 'inventée' en France par les médecins et les pédagogues comme période critique, puis étendue, des garçons pubères bourgeois collégiens, aux filles et aux ouvriers, à une époque où l'encadrement de ce qui est devenue une véritable classe d'âge fait débat (Thiercé 1999).

${ }_{12}$ Cf. notamment Lallemand (1977); Oppong. Adaba, Bekombo-Priso et Mogey (éds.) (1978) : Bonnet (1988) : Rivière (1990); Peatrik (1999); Bekombo (2009). 
différences entre les sexes, elles-mêmes fortement redéfinies par le projet colonial $^{13}$.

$\mathrm{Si}$ les recherches anthropologiques permettent désormais de mieux cerner les étapes sociales et symboliques de l'enfance et leurs variations contextuelles en Afrique, la manière dont ces constructions sociales, et notamment les relations (grand-)parents-enfants, ont pu être affectées historiquement reste encore extrêmement peu connue (McNee, 2004 ; Alber, 2004). Si la diversité de ces constructions à travers le continent en rend la compréhension globale impossible (Rich, 2010), certaines constructions spécifiques, notamment à partir du XIXè siècle, semblent malgré tout identifiables.

\section{'L'enfant africain' : travailleur, écolier, délinquant?}

Comme en Europe, c'est sans doute à partir du XIXè siècle que la place des enfants dans les sociétés africaines commence à changer fondamentalement ${ }^{14}$, notamment sous l'impact des législations coloniales anti-esclavagistes, des crises alimentaires, de l'augmentation dans certains cas de l'esclavage interne, de la progression de l'islam et du christianisme, de l'introduction coloniale de l'économie monétaire, du travail forcé et des cultures obligatoires, de la médecine occidentale et de la scolarisation, sans compter les importants efforts entrepris par les pouvoirs coloniaux et postcoloniaux eux-mêmes pour contrôler, (re)définir, transformer et/ou manipuler les constructions de l'enfance africaine (Diptee et Klein, 2010) ${ }^{15}$.

${ }^{13}$ Sur le thème genre et colonialisme en Afrique, voir notamment McClintock (1995); Amadiume (1997); Hunt, Liu et Quataert (éds.) (1997); Oyěwùmí (1997); Clancy-Smith et Gouda (éds.) (1998) : Allman, Geiger, et Musisi (éds.) (2002); Woodward, Hayes et Minkley (éds.) (2002); Hugon (éd.) (2004): Goerg (éd.) (2007); Goerg, Rodet et Vince (éds.) (2007); Coquery-Vidrovitch (éd.) (2007).

$\times{ }^{14}$ En Europe, bien que le sentiment de l'enfance' $/$ soit ancien (Alexandre-Bidon, 1991), et que les philosophes, notamment ceux du XVIIIè siècle, se soient largement intéressés aux enfants, ce n'est qu'au XIXè siècle que l'enfance devient une préoccupation centrale concourant ainsi à en façonner des contours et un contenu inédits en le construisant comme véritable catégorie sociale (Ariès, [1960] 1973). L'enfant émerge comme un être à protéger, à éduquer ou à remettre sur le droit chemin (ouverture des salles d'asile en France, Kindergärten en Allemagne, école obligatoire, mouvement de lutte contre le travail des enfants et de protection des orphelins, , etc.), mission dont l'Etat se chargera en se substituant peu à peu à l'Eglise qui poursuivra certaines de ses actions parallèlement. Mais l'avènement de cette figure inversée de l'enfant exploité, souvent associée à tort à la seule industrialisation, ne rompt cependant pas avec le travail des enfants qui occupe encore au XIXè siècle tant la sphère paysanne qu'artisanale et domestique. C contemporaine de l'enfant et du migrant africain, d'autres éléments tels que l'esclavage et l'organisation du travail précolonial n'en ont pas moins été déterminants. Il est difficile d'évaluer dans quelle mesure la période coloniale a plutôt représenté une rupture ou une continuité dans ces constructions, il n'en demeure pas moins que la période post-coloniale, 
Si l'on prend l'exemple des colonies frangases, le volet du projet colonial concernant les enfants a été inspir\& suite à l'abolition de l'esclavage interne (1848 et 1905), notamment dans le cadre de sa "mission civilisatrice" (Conklin, 1997), par la nécessaire réinsertion sociale de l'enfance déshéritée au moyen du travail (Thioub, 2003 ; Faye, 2003), mais également par la lutte contre les classes "dangereuses" menée en Europe à la même époque (Rollet, 2001), les anciens esclaves devenant potentiellement des nouveaux prolétaires particulièrement enclins au vagabondage et au crime (Rodet, 2009). Les enfants affranchis sont ainsi placés comme apprentis chez les particuliers ou dans des cuvres de bienfaisance, tandis que les 'délinquants' sont envoyés dans des colonies pénitentières de rééducation par le travail. Dans les deux cas, tutelle et patronage ou colonies pénitentiaires permettent à l'administration comme à l'élite locale de disposer d'une main-d'œuvre nombreuse, quasiment gratuite et corvéable à merci, situation qui ne diffère que peu finalement de l'esclavage qu'ils connaissaient auparavant (Diop, 2003; Thioub, 2003; Ba, 2009; Moitt, 2011).

La fin de l'esclavage rend donc d'une certaine manière le travail des enfants et la circulation de la main-d'œuvre infantile plus visible (Rodet dans ce numéro), d'autant que celle-ci est soutenue par une économie coloniale qui y recourt systématiquement sur ses chantiers de travail forcé ${ }^{16}$.

Il semblerait cependant que l'assistance à l'enfance prenne largement X le pas au XXè siècle sur le répressif (Faye, 2003) avec un intérêt croissant de l'administration pour le vécu des enfants. Mais celui-ci est principalement déployé à travers des programmes de surveillance et d'éducation à la santé des mères (McNee, 2004). Cette approche est notamment motivée par une politique coloniale pro-nataliste qui considère surtout les enfants colonisés comme une future source de main-d'œuvre (Rogers, 1980). Il est ainsi créé en 1939, à Rufisque (Sénégal), avec pour but d'étendre cette expérience à l'ensemble de l'Afrique occidentale française, un service social qui doit répondre à "la préoccupation de l'administration française d'accroître la population noire de cette région, principalement en luttant contre la mortalité infantile" (Rodet, 2009). Il s'agit donc avant tout de préserver le "capital humain" sur lequel repose toute la mise en valeur coloniale (Ekanza, 1997).

en se réclamant de schémas culturels précoloniaux, a également joué un rôle primordial dans la réappropriation et redéfinition de discours tant précoloniaux que coloniaux dans ce domaine.

${ }^{16}$ Sur le recrutement des enfants sur les chantiers coloniaux de travail forcé au Soudan français, voir Rodet (2006). 
Cette préoccupation coloniale pour l'enfance s'étendra ensuite, notamment sous la pression métropolitaine et internationale, à la question du travail des enfants ${ }^{17}$. D'après Hashim et Thorsen (2011), entre 1919 et if 1998, dix conventions furent passées sur le travail des enfants par l'Xoffice International du Travail.

La scolarisation est également au cœur des grands enjeux coloniaux et postcoloniaux concernant l'enfance (Lange, 2001; Lange, éd., 2003). En Europe, la mise en place au XXè siècle de l'école obligatoire jusqu'à douze ans puis quatorze ans, ainsi que les remaniements des cycles scolaires, dans une alchimie de psychologie, de pédagogie, de médecine et de politique (notamment au regard de l'âge de la majorité) participeront de l'établissement de canons européens universalisants. Ces derniers sont imposés aux populations colonisées puis en partie réappropriéfs par cellesci, à partir du système scolaire colonial et post-colonial et des législations des Etats africains au lendemain des Indépendances. C'est sur ces mêmes canons que les organismes internationaux et les ONG construiront une véritable 'culture du développement' et baseront leurs interventions et leurs programmes à destination des enfants.

Ainsi, conjuguées à la lutte contre le travail des enfants, ces politiques définissent en priorité la place de l'enfant en famille ou à l'école, limitant la mobilité enfantine dite 'normale' à ces deux pôles: soit l'enfant suit ses parents migrants soit il migre pour poursuivre sa scolarité (Hashim et Thorsen, 2011 ; Lord, 2011). Toute migration ayant lieu en dehors de ce cadre est dès lors appréhendée comme deviante. Le postulat sur lequel repose cette vision de la mobilité enfantine peut être résumé en ces termes :

(...) it is assumed in western societies that children have a natural need for stability and security which can be provided by the domestic and familial environment. This has resulted in much research and policy towards children being underlaid by assumptions that associate ideal modern childhoods with residential fixity and domestication

(Ní Laoire, Carpena-Méndez, Tyrrell et White, 2010).

Cependant, les taux de scolarisation ne sont pas uniformes en Afrique (87\% en Afrique du Sud et au Lesotho contre $36 \%$ au Tchad et $22 \%$ en Somalie en 2006 d'après l'UNICEF). De plus, pour les enfants qui vont à l'école, le travail au sein et à l'extérieur de la sphère familiale est toujours de mise et l'articulation entre les deux activités se pose différemment selon qu'on adopte le point de vue des enfants eux-mêmes, de leur entourage ou

\footnotetext{
${ }^{17}$ En Afrique occidentale francaise est publié en 1936 un décret portant réglementation du travail des femmes et des enfants en Afrique occidentale française. C'est la première fois qu'une loi métropolitaine sur le travail des femmes et des enfants est en partie retransmise dans le droit colonial en AOF. Le but principal de cette législation était de protéger la femme lors de la gestation, mais surtout de protéger le bébé, et de limiter le travail des enfants (Rodet, 2006). Cf. également Wouango (sous presse).
} 
des ONG qui prolifèrent à partir des années 1970 et organismes internationaux. Dans ce sens, l'éducation mérite d'être interrogée en contexte (Wouango, 2011) et la promotion de l'enfant écolier ne peut faire l'économie d'une réflexion sur le 'droit à l'éducation' et son universalité (Pilon, Martin et Carry, 2010).

Prenant déjà conscience de la mobilité importante des enfants dans les années 1950-1960, suite notamment à l'accélération de l'urbanisation, les

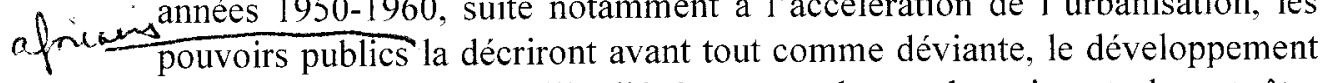
d'une voyoucratie en ville lié à un exode rural croissant devant être combattuq à tout prix (County, 2004). La question de l'enfance délinquante fut certes présente tout au long du XXè siècle posant notamment des problèmes spécifiques en termes de définition de l'enfance, de la délinquance, du rôle des parents dans l'éducation, des questions de genre (voir notamment l'affaire Léonie Gueye analysée par Konaté, 2007). Mais dans les deux décennies qui suivent la Seconde Guerre Mondiale, alors que l'on assiste à la politisation croissante des populations dans le cadre des luttes pour les Indépendances et de la construction du nationalisme err Afre, appuyée par la mise en place systématique de politiques pronatalistes et de protection maternelle (Terretta, 2007), on semble passer clairement, comme en Europe à la même époque (Nagels et Rea, 2007), du paradigme de l'enfance à canaliser au sein des 'classes dangereuses' au XIXè siècle (Grier, 2004) à celui de l'adolescence (ou jeunesse) 'rebelle' du XXè siècle ${ }^{18}$. Ce n'est d'ailleurs sans doute pas un hasard si cette catégorie de la 'jeunesse africaine' émerge alors que les pouvoirs coloniaux euxmêmes considèrent toujours les colonisés comme de 'grands enfants' à éduquer (Cohen, 1970 ; McNee, 2004). La jeunesse africaine postcoloniale sera également au cour des discours politiques sur la Nation en construction (Diouf et Collignon, 2001), les pouvoirs autoritaires faisant l'apologie des enfants de la Nation tout en contrôlant étroitement leur jeunesse alors que celle-ci lutte de plus en plus pour son émancipation politique.

\section{La double invisibilité des enfants migrants africains}

Aux grands bouleversements du XXè siècle précédemment évoqués, et aux conséquences majeurs sur la place et le statut de l'enfant (abolition de l'esclavage, guerres, changement de statut des femmes, du mariage et de la fécondité, décolonisation, crisejéconomiqugs, plus récemment, dévaluation du FCFA, droits de l'enfant, etc.), s'ajoute une réalité démographique

\footnotetext{
${ }^{18}$ La 'jeunesse' finira par apparaître comme politiquement 'explosive' à nombre de gouvernements autoritaires postcoloniaux, ce qui les amènera à reprendre certains schémas coloniaux de criminalisation de celle-ci ou à en mettre de nouveaux en place (De Waal et Argenti, 2002).
} 
incontournable qui devrait rendre de fait les enfants migrants plus visibles que jamais en ce début de XXIè siècle. Or, paradoxalement, ces enfants sont encore le plus souvent appréhendés par les politiques et le monde académique, non pas comme des migrants à part entière, mais plutôt comme des victimes des affres de la globalisation (enfants des rues, enfants soldats, enfants traffiqués, prostitués, réfugiés) ${ }^{19}$ tandis que les 'jeunes', une catégorie floue et difficilement 'contrôlable', composeraient la vaste majorité des migrants illégaux transnationaux ${ }^{20}$. Parmi ces jeunes, les 'mineurs non accompagnés', des individus de moins de dix-huit ans (critère d'âge légal de la majorité dans de nombreux pays d'Europe) sans représentant légal sont devenus des 'mineurs isolés' sur le plan du droit dans les années 1990. Ces 'enfants', qui défient les Etats-Nations et leurs frontières, ont peu à peu été construits en 'problème social' et politique du fait de leur double statut problématique de mineur et d'étranger (Etiemble $2004 \mathrm{a}$ et $b)^{21}$. De toute évidence, les enfants mobiles, nomades ou sans domicile, dont les enfants migrants font partie, déclenchent ce que Ní Laoire, Carpena-Méndez, Naomi Tyrrell et White (2010) désignent comme des moral panics. En n'occupant pas de manière 'adéquate' - notamment en matière de frontières et d'accompagnement - les espaces qui leurf sont dévolus, ils se jouent du contrôle serré des déplacements des mineurs qui est l'apanage de nos societés. des enfeurts

Cette invisibilité sociale se double d'une confusion. En effet, dans la littérature contemporaine sur les enfants migrants en Afrique, c'est la notion de jeunesse (le plus souvent dans son acception etic), très en vogue, qui domine pour appréhender une population migrante non adulte, mais regroupant de fait jeunefet enfants. Si la volonté de les différencier apparaît

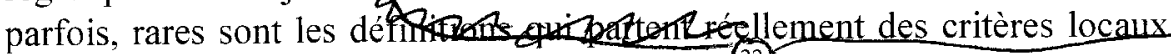
(De Boeck et Honwana, 2005 ; Howana, 2005 22 et qui donnent, même de

\footnotetext{
${ }^{19}$ Comme l'analysent Hashim et Thorsen (2011), les instances internationales constatent à la fin des années 1990 que le débat sur le travail des enfants est dans l'impasse, les pays ne réussissant pas à se mettre d'accord. Désormais, l'attention est tournée exclusivement vers les pires formes de migration et de travail, car le consensus international quant à la dangerosité de ces pratiques et la grave exploitation des enfants semblent plus claires.

${ }^{20}$ A propos des aspirations des jeunes migrants africains, $c f$. Schmitz (2008) et pour une étude de cas Mazzocchetti (2011).

${ }^{21}$ Le numéro 1251 de la revue Honmes et Migrations (2004) est entièrement consacré à la thématique, peu abordée par ailleurs dans les revues scientifiques.

${ }^{22}$ Ungruhe (2010) en donne la définition suivante : the terms youths, adolescents, juveniles and young men (and women) refer to a group of people who are considered to be above what is culturally regarded as being children but who have not yet achieved the social position of an adult in their society (...) youth is neither just a life stage of transition nor a cultural entity in itself but a fluid category integrated in social structures as well as filled with meaning by the young generation's attitudes and actions (...). Therefore, youth is a category that may
} 
manière approximative, les âges ou tranches d'âge auxquels ils correspondent ou encore de simples repères en termes de rôles ou de responsabilités attendues. Or, le continuum artificiel (à la lecture les enfants

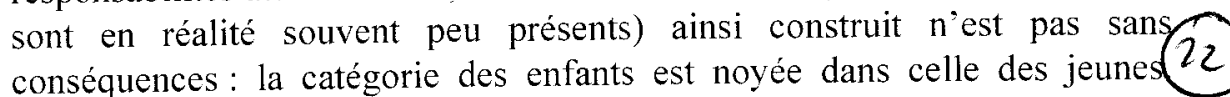
Pourvus d'agency (voir infra.), notion également très en vogue, les enfants, lorsqu'ils apparaissent, sont assimilés aux jeunesy et parés des mêmes modes de revendication ou d'expression que leur aînés! Les spécificités des enfants, parce qu'elles demeurent impensées, ne peuvent alors pas être étudiées.

Cette orientation majoritaire influe sur la définition actuelle de l'enfance en Afrique (De Waal et Argenti, 2002) et rend les enfants migrants d'autant plus invisibles que la production scientifique sur la jeunesse coloniale et postcoloniale en Afrique est abandondante ${ }^{26}$ alors même que l'enfance, et encore plus la petite enfance, dont les contours ne sont pas toujours clairement définis faute d'articulation entre critères locau $x^{27}$ et critères à caractère universalisant, restent tout aussi floues, tant à l'heure actuelle que d'un point de vue historique.

include people from a rather wide range of ages. In the context of juvenile migration, $(\ldots)$ the age bracket of youth between 10 and 32 years (...).

${ }_{23}^{3}$ Alors même qu 'il s'agit généralement dans ces recherches de rompre avec la notion d'êtres en devenir pour se focaliser sur ce qu'ils sont et ce qu'ils font.

${ }^{26}$ Voir notamment: d'Almeida-Topor et Goerg. éds. (1989): Beinart (1991); AlmeidaTopor, Coquery-Vidrovitch et Goerg, éds. (1992); Heap (1997); Comaroff \& Comaroff (2000); Diouf et Collignon, éds. (2001); Glaser (2000 et 2005): De Waal and Argenti, éds. (2002): Burgess (2002); Parsons (2004); Falola, (éd.) (2004); De Boeck et Honwana (2005): Burton (2005); Waller (2006); Fourchard (2006); Christiansen, Utas et Vigh, éds. (2006) ; Bagnol, Matebeni, Simon. Blaser, Manuel et Moutinho (2010).

${ }^{27}$ Les anthropologues, et plus particulièrement les anthropologues de l'enfance, se sont attachés à mettre au jour la manière dont les sociétés étudiées découpent le continuum de la vie et les nombreux travaux montrent autant de similitudes que de différences entre sociétés africaines elles-mêmes et sociétés africaines et sociétés européennes (Lallemand et Le Moal, 1981 ; Lallemand, 1997 ; Rabain, [1979] 1994 ; Baroin, Van Beek, Roulon-Doko et Vincent (éds.), 2003 ; Gottlieb, 2004). 
On peut se demander si les confusions et atermoiements qui agitent les sociétés occidentales quant à leurs propres enfants et jeunes ${ }^{28}$ ne servent pas également trop souvent, sans réflexivité aucune, de prisme à travers lequel les bouleversements des sociétés africaines sont lus? Si l'argument de la globalisation peut expliquer certaines conjonctions de crise (modèles véhiculés par les médias, par les organismes internationaux et les ONG, etc.), il ne peut être invoqué pour éviter la réflexion sur les critères $\widehat{\text { tr }}$ âge et de génération locaux au risque de reproduire une forme de violence néocoloniale sous couvert de protection des enfants.

Certains chercheurs ont ainsi encore tendance à recourir à la construction occidentale de l'enfance au XXè siècle pour analyser ce que vivent les enfants africains (Grier, 2004 ; McNee, 2004 ; Hashim et Thorsen 2011). Si l'on semble être passé thématiquement de l'enfance en danger/dangereuse du XIXè siècle à la 'jeunesse dangereuse' du XXè siècle (avec prolongement dans le XXlè), dont le migrant serait l'une des figures emblématiques, force est de constater que ces approches occultent la plupart du temps l'expérience des enfants.

\section{MIGRATION, MOBILITE, CIRCULATION : OU SONT LES ENFANTS ?}

Si les migrations des adultes ont fait l'objet d'une grande attention, celles des femmes et encore plus des enfants, sont longtemps restées dans l'ombre, que celles-ci soient intra- ou extra-africaine (Cordell, Gregory et Piché, 1996; Le Jeune, Piché, Poirier, 2004 ; Rodet, 2009 ; Kuczynski et Razy, 2009 ; Hashim et Thorsen, 2011) ${ }^{29}$.

\section{Une histoire ancienne des migrations en Afrique}

Les chercheurs sur les migrations depuis le XVIIè siècle en Afrique au Sud du Sahara découpent souvent cette longue histoire en trois grandes étapes (Gregory, 1988; Curtin, 1994). La traite des esclaves et les migrations forcées massives qui y sont liées semblent dominer du XVIIè au XVIIIè siècle. Avec l'abolition et la conquête coloniale de l'intérieur de l'Afrique, la littérature se concentre principalement sur la pénétration de l'économie coloniale et les migrations de travail des hommes dans les villes

${ }^{28}$ On observe un allongement et un raccourcissement de certaines périodes allant de la naissance à l'âge adulte (Segalen, 2010), assortis d'un certain flou, même du côté des spécialistes, au sujet de la période de l'adolescence, qui a pourtant acquis une légitimité X. scientifiquely au XXè siècle (Huerre, 2001)

${ }^{29}$ Les deux derniers numéros d'Hommes et Migrations (2009 et 2010) sur les migrations en Afrique sub-saharienne traitent à peine de ces questions malgré l'avancée des recherches des dix dernières années en la matière. 
coloniales qui en serait le corollaire. Avec la fin de la seconde guerre mondiale, puis les indépendances, l'urbanisation semble s'accélérer et les recherches se concentrent dès lors sur l'exode rural, l'explosion démographique en ville amplifiée par les crises alimentaires des années 1970-1980, puis sur les migrations extra-continentales, notamment vers l'Europe et l'Amérique du Nord.

Cette chronologie, qui peut paraître très grossière, permet néanmoins de dégager plusieurs figures importantes de l'enfant migrant dans la littérature scientifique : l'enfant esclave et, dans sa terminologie contemporaine, l'enfant 'traffiqué', l'enfant travailleur, l'enfant confié, l'enfant du regroupement familial en Europe.

Elle montre également que la migration s'avère un phénomène structurel ancien dans bien des sociétés africaines (aux époques précoloniale $^{30}$ comme coloniale $^{31}$ et est donc considérée en général de manière positive par ses membres. Dans ce cadre, la migration des enfants peut faire partie des options envisagées par les parents ${ }^{32}$ pour leurs enfants afin que ceux-ci augmentent leurs chances de succès dans la vie ${ }^{33}$. Cependant, la question de la mobilité sociale appliquée aux enfants n'est pas si simple. Ainsi, Lallemand (1993) montre que 1'hypothèse soutenue par Goody (1982) selon laquelle les circulations enfantines contemporaines seraient nécessairement liées à la volonté d'ascension sociale des parents est quelque peu réductrice, car elle concerne souvent une période et une situation particulière, l'exode rural, et plus souvent des 'pré-adolescents' et des 'jeunes célibataires' que des 'enfants'.

Parmi les figures de l'enfant africain, celle de l'enfant migrant est aujourd'hui encore peu représentée dans sa diversité et deux pôles en sont généralement privilégiés : d'un côté les enfants de la traite en Afrique et de l'autre, les descendants d'immigrés africains en Europe (Barou, Chappart et Lecadet dans ce numéro). A la jonction entre ces univers sont plus récemment apparus les enfants de l'adoption internationale dont le texte de Getahun restitue, pour le contexte éthiopien, la construction du statut et les

\footnotetext{
${ }^{30}$ Sur le continent africain avant la colonisation, les migrations étaient la condition même de reproduction du système, qu“il s'agisse des échanges commerciaux, de la recherche de nouvelles terres. de la résolution de conflits internes ou de la destinée des cadets. Pour la période coloniale et post-coloniale, les migrations, si elles ont changé de cadre, n'en sont pas moins demeurées une constante dans l'organisation sociale.

${ }^{31}$ Les migrations enfantines, souvent présentées comme marginales en Europe, ne l'ont cependant pas été à toutes les époques dans toutes les classes sociales (Rollet, 2001).

${ }^{32}$ Ces parents sont eux-mêmes souvent des migrants ou ont été des enfants migrants.

${ }^{33}$ Même si dans certains discours, les parents et les autorités la condamnent, on s'aperçoit que dans la pratique peu de personnes au sein des communautés comprennent que l'on puisse tenter d'empêcher des enfants de 14 ans de migrer pour trouver du travail (Dougnon, 2011).
} 
tribulations étatiques; en outre, la recherche accorde de plus en plus d'importance aux enfants left behind (Kane, 2002). Les enjeux éducatifs et la confrontation des systèmes en migration ne peuvent en effet être limités au pays d'accueil. La migration est, comme déjà souligné précédemment, un processus qui relie des espaces multiples, peu en contact direct dans le passé, mais qui le sont désormais de manière quasi-permanente grâce au développement des technologies de communication et des moyens de transports.

\section{Entre domination du modèle migratoire masculin et agency}

Dans les travaux sur les migrations enfantines en Afrique, comme pour les femmes, les migrations des enfants ne sont encore que trop souvent abordées en termes d'émancipation (Rodet, 2009) et de 'chemin vers l'indépendance et la maturité', l'intérêt d'étudier ces migrations étant d'en analyser les bienfaits en termes de mobilité sociale. Les chercheurs examinent ainsi en priorité les migrations 'indépendantes" ${ }^{, 34}$ qui permettraient de mesurer plus précisément la mobilité sociale sans pour autant interroger ces notions. En se concentrant principalement sur les migrations dites indépendantes, nombre de ces études continuent implicitement à considérer le concept de migration de travail - et ses avatars telle que l'exploitation - comme le référent central, neutre et universel pour l'analyse des migrations, alors que ce dernier n'est finalement qu'un référent 'adulte masculin' (Rodet, 2009) ${ }^{35}$. Toute migration s'éloignant de ce modèle, notamment celle des enfants avec leur famille, demeure quasiinvisibles, notamment sur le plan statistique (Pilon et Deleigne). Il en va de même de l'approche en termes de 'famille transnationale' qui a longtemps laissé dans l'ombre des acteurs marginaux, les femmes et surtout les enfants confinés dans la sphère privée (Orellana, Thorne, Chee et Lam, 2001 ; Razy et Baby-Collin, éds., 2011). Pourtant, la grande majorité des migrations dans l'enfance ont précisément lieu dans le cadre de la parenté ou de relations de pseudo-parenté. De même, lorsque les enfants migrent sans leur parents ou apparentés, la décision du départ est approuvée ou connue dans la plupart des cas par au moins l'un des parents ou un membre de l'entourage (Castaldo, Tagoe, Anarfi et Kwankye, 2009). Cette migration renforce dans certains cas leur propre position dans la famille et la communauté - qu'il s'agisse d'enfants en bas âge ou d'enfants plus âgés, ce qui correspond finalement peu aux modèles souvent véhiculés d'enfants

\footnotetext{
${ }^{34}$ Selon Thorsen et Hashim (2011), les migrations indépendantes des enfants sont celles qui s'effectuent sans les parents.

${ }^{35}$ Le modèle migratoire masculin a lui-même subi des changements. notamment au regard des figures du migrant tour à tour travailleur, acteur.
} 
migrants (mais de facto dans ces études plutôt de jeunes) en quête $\mathrm{d}$ 'indépendance, en rupture ou en concurrence avec les adultes (Timera, 2001 ; Rich, 2010 ; Thorsen, 2007 ; Ungruhe, 2010) ${ }^{36}$.

Comme pour les femmes migrantes (Rodet, 2009), vouloir à tout prix démontrer que les enfants sont actifs et indépendants dans la migration, c'est continuer à se référer implicitement à la même dichotomie qui a contribué à rendre ces migrations invisibles. L'utilisation de cette dichotomie implique la définition d'un critère qui permet de déterminer à partir de quel moment la migration peut être considérée comme 'active'. Le critère souvent retenu est le niveau de prise de décision. Or le processus décisionnel s'avère bien plus complexe que ce que pourrait laisser supposer une telle dichotomie, à savoir si le migrant décide lui-même ou non de migrer $^{37}$. Une focalisation sur le niveau de décision dans la migration risque donc d'entraîner la mise à l'écart d'une part importante des migrations, les raisons et causes de ces migrations pouvant être multiples, imbriquées et évolutives pour le sujet lui-même ${ }^{38}$. Les migrants et leur famille ne peuvent pas toujours se rappeler ou connaître tous les facteurs ayant influencé la décision de migrer. Les enfants expérimentent comme les adultes une variété de trajectoires migratoires, souvent de manière combinée à l'échelle de plusieurs années qu'il convient d'analyser sous la forme d'un processus : migrations rurales, urbaines, forcées, saisonnières, définitives... L'adoption d'une définition 'totale' de la migration démontre notamment les interconnexions et le flou des frontières entre ces différentes formes de migration (Rodet, 2009).

De plus, en tentant de montrer que les enfants sont également actifs dans la migration, l'utilisation de cette dichotomie s'avère naturaliste et essentialiste. Elle consiste à traiter les enfants comme un groupe spéfique homogène ignorant les différences au sein de ce même groupe (James et Prout, éds., 1990).

Il est important ici de déconstruire cette dichotomie pour montrer le niveau hiérarchique implicite qu'elle contient (Derrida, 1981). Ainsi, le référent ultime qui permet, dans cette dichotomie, de déterminer si la

${ }^{36}$ De nombreuses études plaquent ainsi certains conflits générationels vécus par les jeunes les ladets sociaur la enfants migrants. Pour une analyse historique de la notion de jeunesse rebelle dans l'Afrique coloniale, voir Waller (2006).

${ }_{37}$ Dans le cas par exemple des migrations forcées des enfants résultant de la mise en esclavage ou du travail forcé colonial pour pallier le manque de main-d'œuvre sur les chantiers coloniaux, la question du niveau de décision apparaît inadaptée.

${ }^{38}$ Massart (2009) recense les raisons et motivations de la migration comme suit pour des "enfants et jeunes ouest-africains mobiles" : "Argent-liquidités", "Biens", "Transformer la réalité sociale", "Fuir un milieu stigmatisé", "Changer de cadre économique", "Les responsabilités familiales", "S'émanciper, s"éduquer", "Mariage des filles". 
migration doit être considérée comme active ou passive, est en réalité la 'migration adulte de travail'. Les migrations des enfants ne sont finalement prises en compte que si elles se rapprochent des critères d'analyse des migrations adultes de travail, c'est-à-dire si les enfants migrent de manière 'indépendante' et temporaire, dans le cadre de la recherche d'un emploi rémunéré en ville. Toute migration s'éloignant de ces critères, comme les migrations dites 'd'accompagnement' ou les migrations rurales, restent, de fait, ignorées. En ne remettant pas en cause cette dichotomie 'migration active/passive', les chercheurs continuent donc implicitement à considérer le concept de migration de travail comme le référent central, neutre et universel dans l'analyse des migrations, alors que ce dernier est finalement un référent 'adulte masculin' qui traduit un point de vue occidental néolibéral, qui plus est 'adultocentrique, 39 .

Si les travaux sur les migrations masculines adultes n'ont pas d'emblée intégré la variable de l'agency, il semblerait que cette notion ait directement été associée au traitement des migrations des enfants. Mais que recouvre le terme agency ${ }^{40}$ dont on peut associer l'émergence, pour le cas qui nous intéresse, à la distanciation des "models of children and childhood with more structural and chronological substrates" (Bluebond-Langner et Korbin, 2007) ? Concernant les études sur l'enfance, on peut distinguer, sans entrer dans une analyse complète, deux sources principales qui ont inspiré la notion d'agency dans un contexte historique où, faut-il le rappeler, la × convention des droits de l'enfant était en pleine élaborations la source la plus souvent mentionnée l'associe à la 'découverte' du caractère historiquement et socialement construit de l'enfance à partir duquel les Children Studies se sont développées en mettant l'accent sur les enfants, non plus être en devenir, mais "êtres actuels" (James et Prout, éds., 1990) dont la voix doit être entendue et écoutée pour elle-même. La seconde source est quant à elle ancrée dans les recherches en psychologie et en psychanalyse qui ont démontré que l'enfant, dès les débuts de sa vie, jouait un rôle d'acteur des interactions sociales ${ }^{41}$. Enfin, la notion d'agency ne

\footnotetext{
${ }^{39}$ De même, l'utilisation de la notion de retour pour des enfants de migrants se rendant dans le pays de leurs parents montre la prégnance du modèle migratoire masculin (Razy 2006).

${ }^{40}$ Chauveau (2005) constate que la notion de social agency est le plus souvent détachée du contexte historique et structurel Dans les travaux sur les jeunes ruraux africains, par ailleurs moins étudiés que leurs homologues urbains, ce même auteur note : Wa capacité des jeunes à se constituer des marges de manœuvre et à contourner ou peser sur les dynamiques sociales qui contribuent à les exclure ne prend sens que par rapport aux aspects d'ordre structurel qui, selon une trajectoire historique particulière, contraignent et conditionnent leur capacité d'action

${ }^{41}$ Notons au passage, que dans de nombreuses sociétés, le petit enfant (généralement avant le sevrage) est dotée, pour des raisons notamment religieuses, de ce qui pourrait être rapprochée d'une forme d'agency (Razy, sous presse).
} 
peut être dissociée de celle d'empowerment qui est au cour des programmes destinés aux populations 'pauvres' ou 'défavorisées' tant au Nord qu'au Sud, marquant son ancrage dans la sphère politique.

Mais dans la littérature actuelle sur les enfants, force est de constater qu'il n'existe pas de consensus autour de la notion d'agency; plus encore, elle est le plus souvent postulée sans être interrogée ni même définie ${ }^{42}$, bien que sa complexité et ses ambiguités puissent être analysées en contexte (Honwana et De Boeck, 2005) ${ }^{43}$. Lorsqu'elle est définie, c'est le plus souvent en puisant dans des travaux concernant les jeunes ou en se basant sur des cadres théoriques pensés pour les adultes (on pense ici à Honwana, [2005] qui convoque Giddens et de Certeau pour élaborer l'agency des enfants-soldats comme une tactical agency ${ }^{44}$ ), ce qui aboutit à opérer un glissement problématique. Quoi qu'il en soit: "This shift in positioning of children's voices has led research, policy, and practice to what might be labeled a commandment to listen to the voices of children" (BluebondLangner et Korbin, 2007).

Si certains pointent les précautions à prendre, d'autres vont plus loin en montrant à partir d'exemples les sous-bassements, problèmes éthiques et épistémologiques, et biais soulevés par the agency movement, dénonçant une utilisation aveugle de la notion sans réfléxion préalable (Lancy, à paraitre).

Pour dépasser le modèle structuraliste dominant, sans pour autant évacuer les contraintes structurelles, et interroger de manière critique la notion de migration indépendante, et son corrolaire la notion d'agency, il convient de saisir les migrations enfantines en contexte et d'en restituer la complexité et les multiples déclinaisons.

\section{Les diverses facettes des migrations enfantines}

Les enfants concernés par la migration le sont en réalité de multiples manières et massivement; la voie des migrations emprunte différentes directions (au sein du pays d'origine; pays d'origine/autre pays d'Afrique ou pays d'origine/pays extra-africain et inversement ${ }^{45}$ et peut revêtir des

\footnotetext{
${ }^{42}$ Ce qui est beaucoup moins clair, c'est le degré d'agency, les effets de cette agency, sans parler de la nature même de l'agency (Bluebond-Langner et Korbin, 2007).

${ }^{43}$ Pour une analyse de cette agency dans des contextes difficiles, voir notamment Evers, Notermans et Ommering (éds.), 2011.

44 " By 'tactical agency', I mean a specific type of agency that is devised to cope with the concrete, immediate conditions of their lives in order to maximize the circumstances created by their military and violent environment (Honwana, 2005).

${ }^{45}$ Si l'intérêt politique, relayée par les chercheurs, pour les questions liées aux migrations vers l'Europe, et en particulier celles impliquant les enfants, dont le caractère est proportionnellement et numériquement marginal par rapport aux migrations intra-africaines,
} 
sens variés, évoluer en raison de la conjoncture, notamment en période de crise (à l'échelle biographique ou collective) :

The powerful ideologies that place idealized childhoods in fixed and bounded spaces are challenged by the complex realities of the lives of many, or most, of the world's children. Research conducted in the global South reveals the ways in which the everyday lives and biographies of many children are marked by mobility, independence and instability.

(Ní Laoire, Carpena-Méndez, Tyrrell et White, 2010).

- Les migrations des enfants - en grande partie sous la forme du fosterage (Goody, 1982 ; Lallemand, 1993; Albert, 2003) très répandu dans les sociétés d'Afrique ${ }^{46}$ - représentent sans doute en Afrique - comme celles des adultes (de Bruijn, van Dijk et Foeken, 2001 ; de Bruijn et Brinkman, 2011) - plutôt la norme que l'exception.

Or, la construction de l'enfant migrant africain contemporain repose encore largement sur deux principes: d'une part, les migrations indépendantes (sous-entendues les 'vraies' migrations) seraient un phénomène récent et d'autre part, la majorité des migrations d'enfants africains s'inscrirait, sinon a priori, souvent a posteriori dans la question du trafic et / ou de l'exploitation à plus ou moins grande échelle ${ }^{48}$.

Or, les migrations des enfants s'appuient de fait très souvent sur des réseaux sociaux et familiaux historiquement ancrés (Rodet, 2009). La

$\propto$ longue histoire des migrations en Afrique inscrit les enfants migrants au sein de contextes culturels et de relations sociales se répartissant entre/sur plusieurs espaces géographiques faisant fi des frontières (Hashim et Thorsen, 2011). Les enfants apparaissent dès lors au cœur des enjeux de translocalite ${ }^{49}$ et de multilocalité (Rodet, 2009).

Resituer les migrations des enfants dans un contexte plus large de $\varnothing$ translocaflité/multilocalité historique rend donc quasiment caduque le

explique en partie le manque de travaux sur ces dernières, il ne faudrait pas qu'un renversement de tendance aboutisse en retour à négliger les circulations enfantines entre Euro-Amérique et Afrique (notamment à double sens. Celles-ci s'inscrivent dans un mouvement similaird (Razy $2007 \mathrm{~b}$ et c). sous peine d'opposer des mouvements migratoires qui nécessitent d'être pensés enjointenent

${ }^{46}$ Lallemand (1993) présente les chiffres de différentes études de cas africains qui montrent de-grandes disparités en matière de confiage: on confie peu ou de manière systématique.

Punusure (1988) en offre un exemple dans la société Sereer Ndut au Sénégal. Lancy (2008) et pour une approche plus ciblée sur les migrations dans l'enfance, Ensor et Gozdziak (éds.) (2010).

49 Sur la notion de translocalité en Afrique, voir notamment Marfaing et Wippel (éds.) (2004). 
modèle de l'enfant migrant seul à la recherche d'un travail, modèle qui renvoie finalement à celui, normatif, de l'enfant apprenti-adulte. De même, ignorer les contextes sociaux et culturels contemporains (notamment les systèmes et les relations de parenté locaux, le statut de l'enfant, etc.) pour ne voir dans les migrations d'enfants africains qu'une conséquence négative de la globalisation, laquelle dénaturerait l'institution 'traditionnelle' du fosterage, ne fait que renforcer ou au mieux qu'occulter plus encore les situations potentiellement à risque dans lesquelles se retrouvent certains enfants $^{50}$.

Une première hypothèse peut être formulée concernant le confiage. Celui-ci peut en effet être envisagé ici comme une forme de cadre, certes plus ou moins lâche, mais incontournable, des migrations enfantines et de leur analyse. Il convient donc en premier lieu d'identifier différentes formes et situations de fosterage qui, sans pour autant recouvrir toutes les formes contemporaines de migrations enfantines, n'en demeurent pas moins un cadre très prégnant (tous les articles du numéro s'y intéressent à des degrés divers) dont il faut restituer l'historicité, comme s'y sont employées Goody (1982) en menant une étude comparative de la circulation des enfants dans plusieurs sociétés d'Afrique de l'Ouest et Lallemand (1993) pour différentes sociétés passées et contemporaines de par le monde, de même que pour les Kotokoli du Centre du Togo (1994) ${ }^{51}$. Rodet (dans ce numéro) illustre le phénomène à une époque charnière où famine et abolition de l'esclavage sont synonymes de manque de main-d'œuvre dans la région de Kayes (Mali), occasionnant fluctuations et brouillage de la frontière entre enfants confiés, petites bonnes et enfants mis en gage. Mieux saisir les contours, la complexité et les déclinaisons contemporaines du confiage, dans ses variantes urbaines flexibles, parfois déliées de la parenté et des liens villageois (Jacquemin), comme la manière dont il est vécu (Lallemand 1994; Notermans, 2008), à travers notamment la notion analytique de résilience ${ }^{52}$ (Porcelli), permet d'une part d'en documenter des pans méconnus et d'identifier des actions, réactions et réflexions proprement enfantines dans les situations de confiage, et d'autre part de mettre au jour d'autres formes éventuelles de migrations enfantines.

\footnotetext{
${ }^{50}$ Les politiques de lutte contre le trafficking peuvent même rendre les enfants concernés encore plus vulnérables en les poussant dans la clandestinité (Massart, 2009) ou encore à se déplacer en petits groupes ou de manière isolée pour ne pas être démasqués lorsque des comités de lutte sont en place. L'un des effets collatéraux de ces politiques est de limiter des déplacements qui, sur un plan social et matériel, bénéficient aux enfants.

${ }^{51}$ Pour une approche comparative de la circulation des enfants, cf. notamment Anthropologie et Sociétés (1988) ; Leblic (éd.) (2004).

${ }^{52}$ Cf. Ensor et Gozdziak (éds.) (2010).
} 
Si en effet, à un moment donné du processus migratoire ou bien tout au long de celui-ci, les déplacements des enfants sont inscrits dans les migrations des adultes, de leur entourage ou dans les décisions des adultes en matière de migration, on peut se demander à chaque fois quelle est la marge de manœuvre des enfants ${ }^{53}$.

Plus encore, aller au-delà dư simple constat d'une situation de confiage nécessite de déplacer la question en analysant la circulation des enfants à partir du statut de l'enfant, des systèmes de parenté et de la parenté pratique comme des autres domaines (économie, politique, religieux, , etc.) dans la société de départ et celle d'arrivée dans une perspective historique et anthropologique, exercice auquel se livre Lallemand (ibid.); il devient alors nécessaire de se demander qui (parents géniteurs ou parents sociaux Lallemand 1976, 2004, mais également enfants) peut remplir auprès de l'enfant les fonctions parentales ${ }^{54}$ telles qu'elles sont localement définies, ce qui peut être très variable. Cela permet de resituer les questions suivantes dans des contextes très localisés : à partir de quel âge les enfants peuvent-ils remplir toutes ou partie de ces fonctions parentales pour eux-mêmes ou auprès d'autres enfants ? Les personnes qui ont en charge un enfant les remplissent-elles? Si tel n'est pas le cas, est-ce acceptable pour l'entourage de l'enfant? La question se pose différemment dans les situations soumises à une conjoncture de crise ( $c f$. les enfants qui deviennent 'chefs de ménage' avec l'épidémie du VIH/Sida, les enfants réfugiés qui sont soumis à des déplacements en temps de guerre ${ }^{55}$ ) où les frontières entre générations deviennent plus floues et les repères moins saisissables.

Quoi qu'il en soit, migrants eux-mêmes ou left behind (Barou), les enfants sont largement concernés par les migrations. En plus de l'identité des accompagnants ou des personnes en charge de l'enfant et des liens de parenté, d'affinité et/ou des relations économiques entretenues entre les 'donneurs' et les 'preneurs' (pour reprendre le vocabulaire de l'alliance), il existe d'autres critères à prendre en compte. Sans vouloir dresser une typologie des enfants migrants, il s'avère indispensable cependant d'attirer l'attention sur la variété et la variabilité des situations et des critères qui

${ }^{53}$ La riche littérature sur les enfants issus de limmigration comme celle concernant la diaspora ne peuvent être analysées ici faute de place.

${ }^{54}$ Goody (1982) introduit la notion de parentalité (parenthood) en déclinant cinq fonctions parentales assumées de manière exclusive soit par les géniteurs soit réparties entre plusieurs individus, apparentés ou non, dans le même lieu ou non: concevoir et/ou engendrer un enfant; l'élever (nourrissage, soins, protection); le former, l'instruire, l'éduquer ; être responsable de ce qu'il fait; le socialiser (nom, statut, droits, devoirs). Godelier (2004) a reformulé et complété cette proposition en y ajoutant l'exercice de l'autorité et l'interdiction de rapports sexuels avec l'enfant (Razy 2010).

${ }_{55}$ Cf. Lange (éd.) (2003) et Boyden et de Berry (éds.) (2004). 
peuvent être déterminants dans la vie de l'enfant migrant et permettre de mieux saisir les spécificités de son itinéraire: le genre, le rang dans la fratrie, l'âge et le statut de l'enfant ${ }^{56}$ et de sa famille au moment du départ, la durée de la migration, en somme, l'histoire migratoire de l'enfant (les phases successives, la récurrence des déplacements de l'enfant qui unscron de ce dernier dans une forme de circulation), les éventuels contacts avec l'entourage qu'il a quitté ; les événements et leurs effets au cours de la migration.

Pour progresser d'avantage dans l'analyse de la catégorie «migrants indépendants" appliquée aux enfants migrants seuls (migrants non accompagnés), il est important d'explorer d'autres facettes problématiques de la définition de 'migrant indépendant' ( $c f$. supra); tout d'abord, de manière générale, considérer la migration comme un processus (Sayad, 1977) permet d'éviter de confondre le voyage, qui peut se faire entièrement ou partiellement seul sans pour autant présager de la suite de celui-ci ; ensuite, être attentif à des formes d'accompagnement diverses, notamment par des parents, des pseudo-parents ou encore des pairs, doit permettre de nuancer la notion de migration indépendante; enfin, selon que l'on parle de migration intra-africaine ou extra-africaine, les critères de qualification des enfants migrants et de leurs déplacements varient.

En dehors des migrations enfantines de crise évoquées plus haut et tout en prenant en compte le fait que les raisons de la migration sont souvent multiples, changeantes et imbriquées, les raisons structurelles des migrations enfantines n'en semblent pas moins relever principalement du travail, de la survie (Einarsdóttir, 2006; voir également Rodet) et de la formation au sens large ${ }^{58}$. Ces causes de la migrations sont rarement dissociables. Elles peuvent également être liées à des enjeux d'alliance ${ }^{59}$ ou à des mises en gage (Lallemand, 1988 et 1993 ; Castle et Diarra, 2003; Razy, 2007 b et c).

Si les déplacements de l'enfant s'inscrivent dans un processus visant à en faire un adulte qui devra jouer différents rôles, notamment celui d'épouse, de mère et de maîtresse de maison pour les filles dans la société,

\footnotetext{
${ }^{56}$ On notera ici que les plus petits qui, lorsqu’ils sont encore allaités, accompagnent le plus souvent leur mère dans ses déplacements pour soins, visites familiales ou dans le cadre d'un projet migratoire passent particulièrement inaperçus...

${ }^{58}$ On ne fait que mentionner ici les migrations enfantines pour raisons médicales qui peuvent être intra- ou extra-africaines.

${ }^{59}$ ll va sans dire que partant des définitions emic de l'enfance, dès lors qu'une fille est mariée, elle n'entre plus dans la catégorie des enfants.

${ }^{61}$ Cf. Lange (éd.) (2003).
} 
il semble que, plus explicitement, "les services presqu'immédiats" (Lallemand, 1993) qu'il peut rendre soient déterminants (Lord, 2011). Considéré comme un futur adulte, c'est bien dans les interstices de ce maillage et dans certains comportements novateurs (De Waal et Argenti, 2002) qu'il faut chercher le degré d'agency des enfants.

Cependant, lorsqu'on évoque l'éducation et l'apprentissag( 61 on pense immédiatement à la scolarisation, généralement dans les écoles offrant un enseignement en français, mais dans les pays musulmans, scolarisation rime 7 également avec formation religieuse dans des medersa (à l'échelle de la ' consession d'un maitre coranique ou d'une structure plus grande et plus formelle) ; l'éducation renvoie également à l'apprentissage de tâches diverses dont domestiques et de compétences notamment culturelles pour les enfants nés à l'étranger (Razy, 2007 b; Gasparetti, 2011 ; Whitehouse, 2009). Le cadre le plus souvent retenu est celui des migrations intraafricaines (intra-régionales notammerit), même si, dans une moindre mesure, les migrations scolaires, puís dans le cadre d'études supérieures dans le sens Afrique-Europe, sont historiquement attestées ${ }^{62}$. Enfin, les migrations dans le sens Europe-Afrique peuvent, contrairement aux apparences ( $c f$. la déscolarisation définitive ou momentanée), également avoir des visées éducatives. Dans ces contextes, le travail des enfants, qui se révèle souvent être au cœur des enjeux migratoires (Grier, 2004 ; Castle et Diarra, 2003; Lord, 2010 et 2011; Hashim et Thorsen, 2011), est généralement une composante de l'éducation des enfants et pas seulement une source de revenu pour l'enfant lui-même et/ou pour son entourage.

Par ailleurs, toute situation de crise -que celle-ci soit «diagnostiquée » par l'enfant lui-même ou par un tiers (structure de prise 7 en charge, membre de l'entourage, etc.) - liée à la situation première de migration, peut générer un autre épisode migratoire, seul ou en groupe. C'est le cas des talibés qui fuient leur maître coranique et rentrent alors chez eux par leurs propres moyens ou deviennent enfants des rues (rapport Húman Rights Watch, 2010) ${ }^{63}$, mais ce peut également être le cas des $\gamma$ petites bonnes (Jacquemin).

Lorsqu'ils franchissent les portes de l'Europe, les migrants âgés de moins de dix-huit ans sont considérés comme des 'mineurs isolés ${ }^{64}$.

\footnotetext{
${ }^{62}$ Principalement pour les élites coloniales et post-coloniales (Bourgoin, 2011).

63 Cf. également Hunt (1993); Mbaye et Fall (1996); Andvig, Canagarajah et Kielland

(2001): Boursin (2002 et 2007); Dieye (2003); Rout Biel (2004): (PERRY (2004); André et

Demonsant (2009). La littérature grise sur les enfants des rues en Afrique est particulièrement abondante, notamment en langue allemande.

${ }^{64}$ A titre d'illustration en termes d'âges, le rapport de Migrations-Etudes note que, pour l'année 2001, les 3/4 des mineurs isolés étrangers recensés ont plus de 15 ans (Etiemble. 2002).

\author{
inninicios $x$
}


L'isolement fait automatiquement entrer ces 'enfants' dans la catégorie des enfants en danger, que celui-ci soit "réel ou potentiel" (Bourgois, 2004). Comme Guillermet (2010) s'y emploie pour la figure coloniale et contemporaine de l'orphelin au Niger en montrant les continuités et discontinuités et leurs effets, il conviendrait de souligner que la seule figure acceptable de l'enfant migrant a été construite progressivement comme celle de l'enfant vulnérable en se focalisant sur les questions liées à la traite - un glissement s'est certes opéré entre travail des enfants et traite, mais c'est surtout la manière d'appréhender les faits qui a changé. De là pourrait être interrogée et levée l'apparente contradiction entre la vision largement partagée de migrations enfantines émancipatrices (appuyée par la notion d'agency), qui ne cadrent pas avec le modèle de l'exploitation, et la figure de l'enfant victime/vulnérable, traitable au niveau du droit.

A ce jour, il ne semble pas exister de modèle migratoire spécifique aux enfants qui pourtant sont très nombreux et présentent certaines caractéristiques; les enfants sont absorbés dans le modèle migratoire masculin adulte qui les projette comme agents émancipés, au même titre que les jeunes, alors que leur réalité est bien plus complexe.

Définis commé vulnérables' mais également dotés d'une capacité d'agir sur ce qui leur arrive (Bluebond-Langner et Korbin, 2007), voilà ce qui ferait la spécificité des enfants. Mais la notion de vulnérabilité pose tout autant de problèmes que celle d'agency, sur laquelle on reviendra, car elle renvoie à une définition de l'enfance qui repose sur un statut de l'enfant et une conception de la famille qui sont loin d'être universels. Cette esquisse de modèle général de l'enfance qui pourrait servir de base à une réflexion sur un modèle migratoire spécifique aux enfants n'est pas totalement satisfaisante. En matière de migration enfantine, on l'a dit, l'accent est mis sur les enfants migrant seuls auxquels est associé précisément le trait de la vulnérabilité ; comme s'il fallait trouver de l'agency dans des formes de résistance et de revendications des enfants pour «contrer» leur vulnérabilité.

Si ce modèle mécanique de l'enfant est pris comme point de départ, mais parvient à être dépassé, il peut amener à réfléchir aux spécificités propres à certains enfants migrants, et peut-être certains enfants migrants africains.

C'est le jeu subtil des constantes et des contraintes qui s'articulent à des histoires uniques de circulations enfantines que les textes du numéro explorent. 


\section{DES HISTOIRES UNIQUES DE MIGRATION ENFANTINE : ENTRE INSTITUTIONS ET PARCOURS INDIVIDUELS}

\section{Des questions transversales}

Revenons succinctement sur certains points déjà évoqués précédemment pour formuler quelques questions.

Tout d'abord, la notion de risque ${ }^{65}$ encouru par l'enfant en situation migratoire apparait dans tous les articles de manière plus ou moins explicite. S'il est clairement démontré aujourd'hui que dans de nombreux cas, ce

1 diagnostic repose souvent sur une méconnaissance des systèmes de parenté et de la parenté pratique dans les sociétés africaines étudiées, en ce qui concerne notamment le partage des fonctions parentales, il ne s'agit cependant pas ici d'en nier l'existence. Ne serait-il pas intéressant d'interroger la notion de risque, et celle de vulnérabilité qui lui est intrinsèquement liée, en relation avec la migration des enfants comme le fait Vidal (2000) à propos du VIH/sida et des femmes lorsqu'il montre l'intrication des risques sociaux et sexuels qui, gouvernés par l'incertitude, n'en demeurent pas moins/soumis à une certaine hiérarchisation pratique (transmission du VIH/sida, positions familiales et statutaires). Dans cette perspective, on pèt se démander ce qui se passerait dans la situation inverse, c'est-à-dire si les enfants ne migraient pas. Ne gagnerait-on pas en
compréhension de ce que vivent les enfants si la question des risques encourus en cas d'immobilité étaient analysés, tant du propre point de vue de l'enfant que de celui de membres de son entourage?

D'autre part, si l'on admet que le confiage, en Afrique comme dans d'autres régions du monde, constitue une institution centrale aux multiples déclinaisons qui sert de cadre, ou de manière moins contraignante parfois de simple trame ${ }^{66}$, à une grande partie des migrations enfantines, la question de ses versions contemporaines 'problématiques' ne peut être évitée (Jacquemin, Porcelli). En effet, qu'il soit question de 'y dévoiement ou encore de 'détournement' de l'institution, le présupposé implicite en est l'existence d'une forme passée régulée souvent appelée 'traditionnelle', en opposition à des dérives actuelles aux conséquences négatives pour les

X. ${ }^{65}$ Porcellifinsouligne notamment à quel point la question du 'bon parent' revient dans les discours des enfants migrants, à savoir si l'enfant est bien nourri, bien habillé, bien chaussé. Mais la relation parent-enfant s'avère également réciproque dans le sens où l'enfant est

$\propto$ considéré comme un producteur qui doit contribuer à l'économie familiale et couvrir dans certains cas une partie de ses besoins personnels (Hashim et/Thorsen 2011). Ce dernier aspect est parfois une des causes de la migration de l'enfant.

${ }^{66}$ Lorsqu'un enfant, qui a grandi dans une société matrilinéaire, quitte le foyer et s'en va de son propre chef rejoindre son oncle maternel dans une autre ville, il inscrit de fait sa migration dans le cadre de référence constitué par le confiage. 
enfants. Là encore, loin de viser à mettre en doute les situations souvent dramatiques vécues par les enfants, ne faudrait-il pas déplacer la question de la différence de nature à la différence de degré ? En d'autres termes, cette institution n'a-t-elle pas en tout temps connu, comme toute institution, ses $y$ *auteurs de troubles et ses profiteurs en tous genres qui, loin de dénature une institution traditionnelle participaient et participent

$\alpha$ encore de la construction dynamique de celle-ci (Bfidet)? La litterature orale en Afrique ne témoigne-t-elle pas de ces situations de confiage malheureuses en nous renseignant sur la chimère d'un âge d'or où tous les enfants confiés vivaient heureux (Argenti, 2010) ? Question de degré donc, qui s'illustrerait dans les crises et changements de tous ordres, notamment dans les crises de subsistance (Lallemand, 1993) - dont la version moderne auraient deux faces: survivre d'un côté et faire du profit de l'autre (Jacquemin, Porcelli), - mais également dans les changements dans les systèmes de parenté et d'alliance, comme dans les relations intergénérationnelles des sociétés lignagères qui redessinent les contours de la famille et de l'éducation (Alber, 2004 ; Notermans, 2004 ; voir également Barou).

L'éducation, au sens large, est au cœur des migrations enfantines, qu'il s'agisse de l'apprentissage d'un métier ou d'activités professionnelles plus ou moins formelles, de tâches domestiques, d'éducation familiale ou scolaire dans des écoles 'françaises' ou coraniques par exemple. Toutes ces formes d'éducation entretiennent souvent des rapports ambigus entre elles (Deleigne et Pilon), mais également avec le travail des enfants dont l'aspect normatif fait partie de l'apprentissage (Hashim et Thorsen, 2011; Lord 2011). Le cadre en est souvent le confiage. L'éducation, notamment scolaire (Deleigne et Pilon), est soumise à de nombreux aléas tant structurels que conjoncturels qui se déclinent souvent entre plusieurs espaces géographiques plus ou moins éloignés socialement et culturellement. Comme le montre Barou, la migration des enfants affecte toute la famille en ce qu'elle contribue notamment à redistribuer les rôles entre générations tant en contexte intra-africain (voir également Alber et Notermans, 2004) qu'en contexte extra-africain.

Mais qui sont les enfants qui habitent ce numéro ? Si la question des tranches d'âge étudiées ou prises en compte est posée dans tous les articles, elle n'en est pas pour autant résolue et doit faire l'objet d'un réel croisement systématique des variables locales, de leur définition et de leur pratique avec celles des institutions et lois locales, des ONG et des organismes internationaux comme des chercheurs (notamment dans les recherches quantitatives). De même, la question du genre, mise en relief dans l'article de Jacquemin, est présente en filigrane dans la plupart des articles. Gageons 
qu'elle sera également au cœur des interrogations sur les migrations enfantines comparant les destinées migratoires des garçons et des filles, sans oublier que le moment du passage de la catégorie d'enfant à adulte est souvent différent selon les sexes.

\section{La voix des enfants}

Force est de constater que les enfants, désormais pourvus d'agency, sont reconnus comme porteurs d'une voix ${ }^{67}$ que les chercheurs auraient, selon certains, trop longtemps négligée ; qu'en est-il réellement?

S'il est vrai que les recherches en anthropologie de l'enfance se sont en grande partie focalisées sur la construction sociale et symbolique de l'enfant à travers le discours des adultes - passage nécessaire s'il en est -, il serait faux d'affirmer qu'aucun des travaux réalisés n'a ménagé de place au point de vue des enfants (Weiss, 1993 et 1995); plus encore, cette 'voix' des enfants est même écoutée et entendue chez les plus petits d'entre eux qui ne parlent pas encore (Bonnet, 1988 ; Lallemand, 1993 ; Rabain, [1979] 1994 ; Gottlieb, 2000 ; Razy, 2007a et 2011 ; Bonnet et Pourchez, 2007, Hardman, 1973).

Comme James (2007) le souligne, des précautions doivent être prises dans l'utilisation de la parole des enfants ${ }^{68}$ car il semble en effet difficile de parler pour et au nom des enfants. Les chercheurs peuvent tout au plus tenter d'être des 'visiteurs' dans leur monde (McNee, 2004). L'une de ces précautions consiste à éviter de tomber dans le travers d'une focalisation sur un type d'acteurs pensés comme isolés; en effet les enfants sont en interaction constante avec les autres acteurs 'parlants' de la société. D'autre part, le chercheur ne peut négliger la construction sociale et symbolique de l'enfant qui modèle ce dernier et sa voix, ni se demander comment cette construction modèle le chercheur lui-même et donc son intervention auprès de l'enfant. Une fois ce cadre général posé, un certain nombre de questions méritent d'être soulevées.

Tout d'abord, envisager d'écouter la voix des enfants, et donc de leur donner la parole, ne relève pas de l'évidence. En effet, la pratique et l'expression des sentiments et des émotions est on ne peut plus variable à

\footnotetext{
${ }^{67}$ Notons au passage que les enfants qui n'ont pas encore acquis le langage (les plus petits) ou qui ne pourront jamais y accéder en raison d'un handicap, en sont de facto dépourvus; dans le cas moins probable où il s'agirait d'une métaphore, son usage nous renseigne sur l'emphase mise sur la communication verbale chez les enfants.

${ }^{68}$ Quand on cite la parole des enfants, il faut notamment prendre en compte la la_nes les problèmes liés aux citations sans réflexivité ni remise en contexte/perspective, de même que la polyphonie des voix, l'intention de l'auteur de la citation et de l'auteur de l'article; il faut enfin se demander à qui sert la citation (James, 2007).
} 
travers l'espace et le temps (Alexandre-Bidon, 1991 ; Guidetti, Lallemand et Morel, éds. 1997; Razy, à paraître). Toutes les sociétés codifient la parole, qui plus est celle sur l'intime et les affects, comme la prise de parole. Celle-ci peut être proscrite ou préscrite en fonction du genre, de la génération et du statut social notamment. Les enfants ne font pas exception comme l'a bien montré Rabain ([1979] 1994) et leur voix ne peut être entendue que si elle est saisie en contexte (Bonnet, 2010 ; voir également Porcelli), ce qui permet de mieux comprendre dans quelle mesure leur voix peut, dans certains cas, être manipulée par les adultes (Chappart et Lecadet, Barou).

L'accès au langage et à la parole, de même, renvoie majoritairement au religieux et la parole peut se révéler dangereuse. Ecouter la voix des enfants dans des sociétés où la norme n'est pas de donner la parole aux enfants (Jacquemin, Porcelli) pose certes des problèmes méthodologiques déjà évoqués par Lallemand à propos du confiage (1994), mais également éthiques, dont Guillermet (2010) résume certains des enjeux en analysant notamment le risque d'un "emploi non réflexif" du principe de "don de la parole aux enfants". Le premier problème identifié découle de qui a été dit plus haut: donner la parole à tous les enfants par décret repose sur une vision universaliste de l'enfance qui fait fi de l'analyse des contextes où sa construction sociale et culturelle n'obéit pas aux canons occidentaux ${ }^{69}$. De manière sous-jacente, on pressent que cette parole donnée est pensée comme nécessairement bénéfique voire libératrice et salvatrice pour l'enfant, principe issu de la psychologie et de la psychanalyse (et visant l'empowerment dans sa version développementistex 'yui, là encore, n'est absolument pas universel. Enfin, et c'est là un point crucial, une fois la parole de l'enfant donnée au chercheur qui quitte son terrain, qu'advient-il de sa parole et qu'advient-il de l'enfant qui, en parlant, a accédé à une forme de prise de conscience de son expérience, voire à un processus de subjectivation - lequel peut devenir bien encombrant? Plus encore, en situation migratoire, d'où parle l'enfant africain ?

Entre participer de la reproduction d'une forme de mutisme des enfants et provoquer leur prise de parole coûte que coûte ou simplement les écouter, la question se pose de savoir si ce sont les précautions et 'innovations' méthodologiques ou encore les codes éthiques qui permettront de résoudre les problèmes soulevés. A qui et à quoi servent les processus de visibilisation des enfants? Dans quelles conditions, pourquoi et comment les enfants, et notamment les enfants migrants africains, apparaissent-ils

\footnotetext{
69 Parmi ses critiques, Lancy (à paraître) qualifie cette démarche d'ethnocentrique, hégémonique, issue d'une classe dominante bien-pensante et montre le caractère contreproductif que peut avoir son utilisation sans réflexivité.
} 
dans les travaux scientifiques et sur les scènes publiques nationale et internationale?

\section{L'enfant-enjeu}

Comme le souligne McNee (2004), dans de nombreuses sociétés africaines, l'enfant a toujours été clairement une source de joie, au cœur de la reproduction sociale et économique, une grande source de main-d'œuvre, et donc potentiellement de richesse dans des sociétés où la production agricole était essentiellement extensive du fait de la pauvreté des sols (d'où l'importance de la dot pour la famille de la fille lors des mariages qui permettait de pallier la perte de main-d'œuvre). Rodet note ainsi que "Les enfants ne sont pas ici seulement considérés comme des 'apprentis-adultes', mais ce sont surtout des membres de la famille à qui sont assignés des tâches spécifiques devant contribuer à l'économie familiale" "70. Leur migration peut notamment être lié à ce rôle de producteur.

Comme le montrent en particulier les textes de Getahun, Barou, Chappart et Lecadet, les enfants migrants africains, sont au cœur d'enjeux politiques, économiques, sociaux, culturels, religieux, scientifiques, légaux nationaux et internationaux (dans les politiques publiques à l'égard des sans-papiers et dans la mise en place des dispositifs d'expulsion, dans les luttes politiques et sociales des parents migrants, dans les conflits d'éducation mais également entre sphère privée et sphère publique, 1. étroitement lié à la question de la gestion de l'enfance dans les familles au sein des Etats-Nations). Ces enjeux résident aussi bien au niveau institutionnel (Etats africains et européens et régimes politiques, organisations internationales et $\mathrm{ONG}$ ) qu'au niveau des populations locales dans les de départ mais également d'arrivée (familles, voisinage, communautés, autorités). Si, ce qui se joue à travers les enfants peut être traduit de manière simplifiée en termes de tensions entre production et reproduction sociale en Afrique, en Europe et entre Afrique et Europe (en matière économique, d'alliance, identitaire, religieuse, etc. $)^{71}$, la prévalence et la morphologie des migrations dites 'indépendantes' entre pays africains, à mettre au tribut d'une certaine grille de lecture et d'une législation n'empêchant pas les déplacements d'enfants ni leur utilisation sur le marché du travail (Deleigne et Pilon), doivent faire l'objet de toutes les attentions des chercheurs.

\footnotetext{
${ }^{70}$ Voir également Iliffe (1987) et Lord (2011)

71 A ce titre, la circulation des enfants peut s inscrire dans le paiement de la dette

1 migratoire une forme de dette communautaire morale, contractée par ses ascendants au moment du départ des femmes, parties rejoindre leur mari à l'étranger (Razy, 2007b).
} 
Toutefois, la diversité des contextes et des expériences migratoires enfantines, tout comme leur complexité, inclinent à revisiter sous un autre angle leur spécificité entre agency et vulnérabilité. Tour à tour ou simultanément objet et sujet, vulnérable mais doté d'agency (BluebondLangner et Korbin, 2007), les enfants, mais plus encore les enfants migrants, présentent la particularité d'être 'sur-investis', ce qui n'est pas, loin s'en faut, un gage de protection. Cet aspect est rarement compris et/ou pris en compte, que ce soit par l'entourage de l'enfant, les institutions ou encore les chercheurs. On a notamment trop longtemps négligé les changements et les adaptations que subissait le double entourage ${ }^{72}$ de l'enfant migrant ${ }^{73}$ : ceux qui remplissent les fonctions parentales changent, mais l'entourage formé par les pairs également, et il ne s'agit pas nécessairement d'une ressource. Comme il est permis de le contaster dans nombre des articles du numéro, l'entourage peut être mal disposé à l'égard de l'enfant (la question ressort plus moins explicitement dans tous les articles). Ce qui est propre aux enfants migrants, c'est précisément ce double entourage issu de la situation de migration et dans lequel les enfants sont inscrits ; ils peuvent y puiser ou tenter d'agir contre lui, le modeler ou le compléter : la quête de ressources humaines servant à remplir l'une ou l'autre des fonctions parentales, même partiellement, même sporadiquement, est fondamentale. L'enfant présente également la faculté, pas nécessairement actualisée de manière linéaire, de composer et parfois mobiliser son double entourage en situation de migration comme de s'en affranchir et de 'jouer' sur plusieurs registres (voir Porcelli, Barou).

Différencier ce qui relève de la structure de ce qui relève de la crise et deviendra peut-être à terme structurel - ne peut aboutir qu'à la condition de démêler les liens historiques et actuels complexes entre migrations enfantines et événements, tant à une échelle collective (au niveau des institutions comme des situations de crises) qu'à une échelle biographique.

Porcelli et Barou, tout comme Ní Laoire, Carpena-Méndez, Tyrrell et White (2010) et Evers (2011), montrent notamment à quel point les relations sociales, les notions d'appartenances et les relations à des lieux spécifiques et la mémoire de ceux-ci s'avèrent fluides, contextuelles, mobiles et largement évolutives.

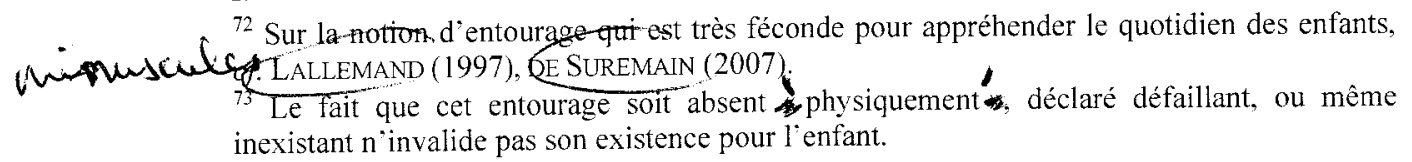
inexistant n'invalide pas son existence pour l'enfant. 


\section{Les articles: une analyse de parcours migratoires dans l'enfance entre institutions locales et insitutions globales}

L'article de Rodet permet tout d'abord de resituer les migrations des enfants en Afrique de l'Ouest, et plus particulièrement au Mali, dans leur dimension historique. L'auteure aborde notamment le caractère socialement et historiquement construit des stratégies migratoires mises en place par les parents pour eux-mêmes et leurs enfants en période de crise, que ceux-ci migrent ensemble ou non. Cet article invite à mieux comprendre le flou des frontières entre enfants confiés et esclavage domestique provoqué par les profonds changements économiques et sociaux que connaît la région de Kayes (Mali) au début du XXè siècle.

L'article de Jacquemin fait écho à celui de Rodet en démontrant que l'institution des 'petites bonnes' en Côte d'Ivoire a connu de nombreuses transformations depuis les années 1980 , notamment sous l'impact de la crise économique et des ajustements structurels. L'auteure souligne à quel point ces changements économiques et sociaux ont en particulier des conséquences genrées sur les migrations dans l'enfance et le travail des enfants.

Comme le souligne l'article de Deleigne et Pilon, la migration peut

$\mathcal{L}$ également avoir des impacts tant positifs que négatifs sur la scolarisation, même si cet aspect demeure complexe à analyser et difficilement quantifiable. Cette approche par la scolarisation, en utilisant notamment les documents de recensement de la population au Burkina Faso, permet de rendre les migrations enfantines visibles d'un point de vue démographique, ce qui a rarement été réalisé jusqu'ici. Même si les travaux dans ce domaine se sont particulièrement développées au Burkina Faso dans les quinze dernières années (voir entre autres Cordell, Gregory et Piché, 1996), ils mériteraient d'être étendus de manière systématique à d'autres pays africains.

L'article de Porcelli, par une approche psychologique qualitative, rend les expériences migratoires des enfants concrètes et analyse de manière précise leur vision de celle-ci. Cette photographie détaillée du fosterage et pseudo-fosterage montre notamment la complexité des configurations au sein de l'institution du confiage et les stratégies multiples de résilience mises en place par les enfants migrants dans leur rapport aux adultes pour se construire un équilibre affectif indispensable à leur cheminement vers l'âge adulte.

Ces questions affectent également les enfants en situation de confiage dans des univers transnationaux. $\mathrm{Si}$, comme le souligne Barou dans son article sur les changements familiaux chez les demandeurs d'asile et les réfugiés en France, la communication entre pays d'origine et pays d'accueil 
peut être coupée en période de crise, notamment de guerre, créant des situations familiales particulièrement douloureuses - les parents migrar $\left[t_{1}^{\prime}, \mathrm{d} d \mathrm{u}\right.$

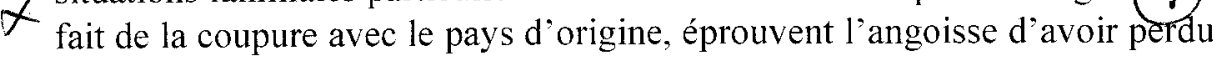
leurs enfants ici comme là-bas - les modèles éducatifs africains comme européens n'en continuent pas moins de circuler et d'affecter les parents comme les enfants, entre rejet, réappropriation et hybridation, ce qui n'est que rarement appréhendé par les services sociaux dans les pays d'accueil.

Les enfants migrants peuvent de ce fait devenir, comme le démontrent Chappart et Lecadet dans leur article sur la place des enfants dans l'évolution des luttes et des pratiques politiques autour des sans-papiers, des enjeux politiques et institutionnels importants qui laissent finalement peu de place au vécu même des enfants dans la migration.

Comme le montre enfin l'article de Getahun sur l'histoire de l'adoption internationale en Ethiopie, ces questions peuvent recouvrir des enjeux politiques internationaux forts, notamment entre pays 'du Nord' et pays 'du Sud', entre institutions locales et institutions globales, la figure médiatrice de l'enfant migrant renseignant en particulier sur les rapports historiques complexes et souvent inégalitaires entre ces différentes entités, même lorsque le rapport colonial historique est de facto absent.

Pour finir, l'ensemble des articles démontrent la nécessité de réfléchir sur des cadres conceptuels et des stratégies de recherches permettant d'améliorer nos connaissances quant à la nature complexe et multiple de l'expérience migratoire africaine dans l'enfance.

Nous espérons, par ce numéro spécial du Journal des Africanistes sur les migrations africaines de et dans l'enfance, ouvrir quelques pistes de réflexion, mais surtout susciter un plus vif intérêt des chercheurs afin qu'ils contribuent, après l'avoir reformulée et revisitée, à mettre à l'ágenda politique une problématique cruciale qui concerne tant les pays du Nord que les pays du Sud.

\section{Références bibliographiques}

Agullar, M. (éd.), 2007, Rethinking Age in Africa, Trenton, Africa World Press.

AlBer E., 2003, Denying Biological Parenthood: Fosterage in Northern Benin, Ethnos 68(4), pp.487-506.

-, 2004, Grandparents as Foster-Parents: Transformations in Foster Relations between Grandparents and Grandchildren in Northern Benin, Africa : Journal of the International African Institute, vol. 74, $\mathrm{n}^{\circ} 1$, Grandparents and Grandchildren, pp.28-46.

ALEXANDRE-BIDON, D. 1991 Grandeur et renaissance du sentiment de l'enfance au Moyen Âge, in J. VERGER, Educations médiévales, L'enfance, l'école, l'Eglise en Occident (VIe-XVe siècles), Histoire de l'Education, n ${ }^{\circ} 50$, pp.39-63. 
Allman, J., S. Geiger, N. Musisi (éds.), 2002, Women in African Colonial Histories, Bloomington \& Indianapolis, Indiana University Press.

D'AlMEIDA-TOPOR, H. et O. GOERG (éds.), 1989, Le mouvement associatif des jeunes en Afrique Noire francophone au XXè siècle, Groupe, cahier n ${ }^{\circ} 12$, Paris, L'Harmattan.

D'Almeida-Topor H., C. COQuery-Vidrovitch, O. Goerg et F. Guitart, 1992, Les Jeunes en Afrique. Tome 1 : Evolution et rôle (XIXe-XXe siècles), Paris, L'Harmattan.

- 1992, Les Jeunes en Afrique. Tome 2: La politique et la ville, Paris, L'Harmattan.

AMADIUME, I., 1997, Reinventing Africa. Matriarchy, Religion \& Culture, London, Zed Books Ltd.

André, P. et J.-L. Demonsant, 2009, Koranic Schools in Senegal : An actual barrier to formal education?, Department of Economics and Finance Working Papers from Universidad de Guanajuato, Department of Economics and Statistics.

Anthropologie et Sociétés, 1988, Les enfants nomades, vol. 12, nº 2.

Andvig, J.-C., S. CANAGARAJAH et A. KiElland, 2001, Issues in Child Labor in Africa, Africa Region Human Development Working Paper Series, The World Bank.

Argenti, N., 2010, Things that Don't Come by the Road: Folktales, Fosterage, and Memories of Slavery in the Cameroon Grassfields, Comparative Studies in Society and History, $\mathrm{n}^{\circ} 52$, pp.224-254.

ARIES, P., [1960] 1973, L'enfant et la vie familiale sous l'Ancien Régime, Paris, Editions du Seuil.

BA, D. C., 2009, France colonial charitable et Enfance indigène affranchie. Une altérité conflictuelle mal assumé 1848-1905, Historiens et Géographes $d u$ Sénégal, $\mathrm{n}^{\circ} 8$, L'esclavage et ses traites en Afrique, discours mémoiels et saviors interdits, pp.46-57.

Bagnol, B., Z. Matebeni, A. Simon, T. M. Blaser, S. Manuel et L. Moutinho, 2010, Transforming Youth Identities: Interactions Across "Races/ Colors/ Ethnicities," Gender, Class, and Sexualities in Johannesburg, South Africa, Sexuality Research and Social Policy, vol. 7, n4, pp.283-297

Baroin, C., W. VAn Beek, P. Roulon-Doko, et J.-F. Vincent (éds.), 2003, L'enfant dans le basin du lac Tchad, Journal des Africanistes, , 72.

BARTHÉLÉMY, P., 2011, Africaines et diplômées à l'époque coloniale, Rennes, Presses Universitaires de Rennes.

BEINART, W., 1991, Transkeian Migrant Workers and Youth Labour on the Natal Sugar Estates 1918-1948, The Journal of African History, 32/1 : 41-63.

Bekombo Priso, M., 2009, Penser l'Afrique. Regards d'un ethnologue dwala. Textes choisis et présentés par A. ADLER, R. VERDIER et M.-D. MOUTON, Nanterre, Société d'ethnologie.

BENOIST (DE), J.-R., 1987, Eglise et pouvoir colonial au Soudan Français. Les relations entre les administrateurs et les missionnaires catholiques dans la Boucle du Niger, de 1885 à 1945, Paris, Karthala.

BLUEBOND-LANGNER M. et J. E. KorBIN, 2007, Challenges and Opportunities in the Anthropology of Childhoods : An Introduction to "Children, Childhoods, 
and Childhood Studies", American Anthropologist, vol. 109, n², pp.241-246

Bonnet, D., 1988, Corps biologique, corps social. Procréation et maladie de l'enfant en pays mossi, Burkina Faso, Paris, Editions de l'ORSTOM.

- 2010, La construction sociale de l'enfance: une variété de normes et de contexte, Informations sociales, 4(160), pp.12-18.

BONNET, D. et L. POURCHEZ (éds.), 2007, Du soin au rite dans l'enfance, Ramonville, Erès \& IRD.

Bonnet, D., Rollet, C., C.-E. de Suremain, (éds.) (sous presse), Modèles d'enfances. Successions, transformations, croisements, Paris, Editions des Archives contemporaines.

BOUCHE, D., 1975, L'enseignement dans les territoires français de I'Afrique occidentale de 1817 à 1920 . Mission civilisatrice ou formation d'une élite ? (2 vol.), These de doctorat, Université Lille III.

Bourgois, L. 2004, Un statut indéfini, des réponses éclatées, Hommes et migrations, $\mathrm{n}^{\circ} 1251$, pp.6-8

Bourgoun, F., 2011, Des individualistes globaux : ruptures et discontinuités dans les familles d'élites africaines transnationales, in E. RAZY et V. BABY-COLLIN (éds.), La famille transnationale dans tous ses états, Autrepart, n57, pp.299314.

BOURSIN, F., 2002, Travail et trafic des enfants versus scolarisation, Colloque sur La recherche face aux défis de l'éducation au Burkina Faso, 19-22 novembre 2002, Ouagadougou, AREB - INSS/CNRST - IRD.

- 2007, Travail et trafic des enfants : le cas du Burkina Faso, in Compaoré et al. (éds), La question éducative au Burkina Faso. Regards pluriels, CNRST, Ouagadougou, pp.161-181.

BOYDEN, J. et J. DE BERRY (éds.), 2004, Children and youth on the front line. Ethnography, armed conflict and displacement, Berghahn Books, New York.

BRIL, B., 1988, Le développenent psychologique est-il universel?, Paris, Presses Universitaires de France.

BRUIJN (DE), M., R. VAN DIJK, et D. FOEKEN, 2001, Mobile Africa: An introduction, in M. DE BRUIJN, R. VAN DIJK et D. FOEKEN (éds.), Mobile Africa : Changing Patterns of Movement in Africa and Beyond, Leiden, Boston et Köln, Brill, pp.1-7.

BRUIJN (DE), M. et I. BRINKMAN, 2011, "Communicating in Africa". Following Mobile Communities in Angola and Cameroon, in E. RAZY et V. BABY-COLLIN (éds.), La famille transnationale dans tous ses états, Autrepart, n ${ }^{\circ} 57$, pp.41-58.

BurTon, A., 2005, African Underclass : Urbanisation, Crime and Colonial Order in Dar-es-Salaam, Oxford, James Currey Publishers.

BURGESS, T., 2002, Cinema, bell bottoms and miniskirts : struggles over youth and citizenship in revolutionary Zanzibar, International Journal of African Historical Studies, 35, pp.287-313

Campbell, G., S. Miers et J.Miller (éds.), 2009, Children in Slavery, Athens, Ohio University Press.

-, 2011. Child slaves in the modern world, Athens, Ohio University Press.

CASTLE, S. et A. DiARRA, 2003, The international migration of young malians : tradition, necessity or rite of passage, Research report, London, London School of Hygiene and Tropical Medicine. 
ChauveaU, J.-P., 2005, Introduction thématique : Les jeunes ruraux à la croisée des chemins, in J.-P CHAUVEAU, (éd.), Afrique et développement, Afrique contemporaine, $\mathrm{n}^{\circ} 214$, pp. 15-35.

Christiansen, C., M. UtAs et H. E. ViGH (éds.), 2006, Navigating youth generating adulthood: social becoming in an African context, Stockholm, Nordic Africa Institute Publ.

Clancy-Smith, J., F. Gouda (éds.), 1998, Domesticating the Empire: Race, Gender, and Family Life in French and Dutch Colonialism, Charlottesville, The University Press of Virginia.

COE, C., 2010, Domestic Violence and Child Circulation in Southeastern Gold Coast, 1905-1928, in E. Burrill, R. ROBErTs et E. THORNBErRy (éds.), Domestic Violence and the Law in Colonial and Postcolonial Africa, Athens, Ohio University Press.

COHEN, W. B., 1970, The Colonized as Child : British and French Colonial Rule, African Historical Studies, vol. 3, n², pp.427-431

Collinson, M., 2008, Possible future directions for work on migration, youth and children, paper prepared for the conference, "Migrant Youth \& Children of Migrants in a Globalized World", 24-26 avril 2008, Bellagio, Italie.

Conklin, A. L., 1997, A Mission to Civilize. The Republican Idea of Empire in France and West Africa, 1895-1930, Stanford, Stanford University Press.

Comaroff, J. et D., 2000, Réflexions sur la jeunesse : du passé à la postcolonie, Politique africaine, 80, pp. 90-110.

COQuery-Vidrovitch, Catherine (éd.), 2007. Les femmes, le droit et la justice, Cahiers d'études africaines, 187-188.

Cordell, D. D., J. W. Gregory et V. Piché, 1996, Hoe and Wage, A Social History of a Circular Migration System in West Africa, Boulder, Westview Press.

COUNTY, B., 2004, "The Real Gangrene of Our Youth": Demographics and the Politicization of Urban Migration during Mali's First Five Year Plan, 19601966, unpublished MA Thesis, Columbia University.

CURTIN, P.D., 1994, Why People Move: Migration in African History, The Sixteenth Charles Edmondson Historical Lectures, 7-8 March 1994, Waco, Texas, Baylor University.

DANIC, I., DElal AndE, J., RAYOU, P., 2006, Enquêter auprès d'enfants et de jeunes. Objets, méthodes et terrains de recherche en sciences sociales, Rennes, Presses universitaires de Rennes.

DE BOECK, F. et A, HonwanA, 2005, Introduction : Children and Youth in Africa. Agency, Identity \& Place, in A. HONwANA et F. DE BOECK, Makers and Breakers. Children and Youth in Postcolonial Africa, James Curey, Oxford, pp. $1-18$

DERrIDA, J., 1981, Positions. Traduit par A. BASS, London, The Athlone Press.

DIENG, M., 2003, Maladies des enfants et initiatives du pouvoir au Sénégal (XIXèXXè siècles), Les cahiers histoire et civilisations, $\mathrm{n}^{\circ} 1$, pp.75-83.

Dougnon, I., 2011, Child Trafficking or Labor Migration?: A Historical Perspective from Mali's Dogon Country, Humanity : An International Journal of Human Rights, Humanitarianism, and Development, vol. 2, n², pp.85-105.

DE WAAL, A. et N. ARGENTI, 2002, Young Africa : realising the rights of children 
and youth, Trenton, Africa World Press.

DiAllo, K., 2003, Organisations syndicales et enfants travailleurs au Sénégal, Les cahiers histoire et civilisations, $\mathrm{n}^{\circ} 1, \mathrm{pp} .105-114$.

DIEYE, M. M., 2003, Les enfants de la rue: symptômes de l'irruption de la violence, Les cahiers histoire et civilisations, $n^{\circ} 1$, pp.131-139.

DioP, B. (éd.), 2003, L'Enfance, Les cahiers histoire et civilisations, n²1, Dakar, UCAD.

Diouf, M. et R. Collignon, 2001, Les jeunes du Sud et le temps du monde: identités, conflits et adaptation, in M. Diouf, et R. CollignON (éds.), Les jeunes, hantise de l'espace public dans les sociétés du Sud ?, Autrepart, n²18, pp.5-15.

DOMENACH, H. et M. PIQUet, 1995, Les Migrations, Paris, PUF (Que sais-je ?).

DUPIRE, M., 1988, L'ambiguiité structurale du fosterage dans uen société matrivirilocale (Sereer Ndut, Sénégal), Anthropologie et Sociétés, 12(2), pp.7-24.

ENSOR, M.O. et GOZDZIAK, E. M., (éds.), 2010, Children and Migration. At the Crossroads of Resiliency and vulnerability, Palgrave Macmillan, Hampshire.

EINARSDÓtTIR, J., 2006, Relocation of Children. Fosterage and child death in Biombo, Guinea-Bissau, in C. Christiansen, M. UTAS et H. E. Vigh (éds.), Navigating Youth, Generating Adulthood: Social Becoming in an African Context, Uppsala, Nordic africa Institute, pp.183-200.

ETIEMBLE, A., 2002, Les mineurs isolés étrangers en France. Evaluation quantitative de la population accueillie à l'Aide Sociale à l'Enfance, les termes de l'accueil et de la prise en charge, Migrations Etudes, $n^{\circ} 109$, Direction de la Population et des Migrations.

-, $2004 a$, Quelle protection pour les mineurs isolés en France, Hommes et migrations, $\mathrm{n}^{\circ} 1251$, pp.9-22.

-, $2004 \mathrm{~b}$, Mineurs isolés demandeurs d'asile, Hommes et migrations, $\mathrm{n}^{\circ} 1251$, pp.51-61.

EKANZA, S. P., 1997, Tendances démographiques et transformations de la société en AOF, in C. BECKER, S. MBAYE et I. THIOUB (éds.), AOF : réalités et héritages. Sociétés ouest-africaines et ordre colonial, 1895-1960, tome 2, Dakar, Direction des Archives du Sénégal, pp.984-994.

EVERS, S.J.T.M., 2011, Kinning in the Imagination: Perceptions of Kinship and Family History among Chagossian Children in Mauritius, in S.J.T.M. EvERS, C. NOTERMANS et E. VAN OMMERING (éds.), Not just a victim: The child as catalyst and witness of contemporary Africa, Leiden, Brill Academic Publishers, pp.79-105.

Evers, S.J.T.M., C. Notermans et E. VAN OMMERING (éds.), 2011, Not just a victim: The child as catalyst and witness of contemporary Africa, Leiden, Brill Academic Publishers.

Falola, T., et P. Love.oy (éds.), 1994, Pawnship in Africa: Debt Bondage in Historical Perspective, Boulder, Lynn Reiner.

FAlOLA, T. (éd.), 2004, Teen Life in Africa, Wesport, Greenwood Press.

FASSIN, D., 2010, La raison humanitaire. Une histoire morale du temps présent, Paris, Seuil/Gallimard.

FAYE, O., 2003, Assister ou punir l'enfant. Quelle expérience pour l'Etat colonial au Sénégal ?, Les cahiers histoire et civilisations, $\mathrm{n}^{\circ} 1$, pp.17-29. 
FOURCHARD, L., 2006, Lagos and the Invention of Juvenile Delinquency in Nigeria, 1920-1960, Journal of African History, 47, pp.115-37.

GASPARETTI, F., 2011, Relying on Teranga : Senegalese Migrants to Italy and Their Children Left Behind, in E. Razy et V. Baby-Collin (éds.), La famille transnationale dans tous ses états, Autrepart, 57, pp.215-232.

GETZ, T., 2011, British Magistrates and Unfree Children in Early Colonial Gold Coast, 1874-1899, in G. CAmpBell, G., S. Miers et J. Miller (éds.), Child slaves in the modern world, Athens, Ohio University Press, pp.157-172.

GLASER, C., 2000, Bo-Tsotsi : The Youth Gangs of Soweto, 1935-1976, Portsmouth, Heinemann.

—, 2005, Managing the sexuality of urban youth: Johannesburg, 1920s-1960s, International Journal of African Historical Studies, 38, pp.301-27.

GOERG, O., M. RODET et N. VINCE N. (éds.), 2007, Fracturing Binarisms : Gender and Colonialisms in Africa, Stichproben, $\mathrm{n}^{\circ} 12$.

GOERG, O. (éd.) 2007, Perspectives historiques sur le genre en Afrique. Cahiers Afrique, n'23 (SEDET-Paris7), Paris, L'Harmattan.

GOODY E. N., 1982, Parenthood and social reproduction. Fostering and occupational roles in West Africa, Cambridge, Cambridge University Press.

GotTLIEB, A. 2004, The Afterlife Is Where We Come From : The Culture of Infancy in West Africa, Chicago, University of Chicago Press.

,- 2000 , Où sont passés tous les bébés ? Vers une anthropologie du nourrisson, in J.-L. JAMARD, E. TERRAY et M. XANTHAKOU (éds.), En substances. Textes pour Françoise Héritier, Paris, Fayard, pp.366-385.

Gregory, J., 1988. Migrations et urbanisation, in D. TABUTIN éd. Population et société en Afrique au Sud du Sahara, L'Harmattan, Paris, pp.369-399.

Grier, B., 2004, Child Labor and Africanist Scholarship: A Critical Overview, African Studies Review, vol. 47, n², pp.1-25.

GrIER, B., 2005, Invisible Hands: Child Labor and the State in Colonial Zimbabwe, Portsmouth, Heinemann.

Guidetti, M., S. LALlEMAND, M.-F. Morel (éds.), 1997, Enfances d'ailleurs, d'hier et d'aujourd'hui, Paris, Armand Colin.

Gulllermet, E., 2010, Constructions de l'orphelin au Niger. Anthropologie d'une enfance globalisée, Sarrebrück, Editions universitaires européennes.

GUYER J. I. and S. M. E. BELINGA, 1995, Wealth in People as Wealth in Knowledge : Accumulation and Composition in Equatorial Africa Journal of African History, 36, $\mathrm{n}^{\circ} 1$, pp.91-120

HARDMAN, C., 1993, Unterwegs zu einer Anthropologie der Kindheit, in M.-J. VAN DE LOO et M. REINHART, Kinder. Ethnologische Forschungen in fünf Kontinenten, München, Trickster Verlag, pp.60-77.

HARDMAN, C. 1973, Can there be an anthropology of children?, Journal of the Anthropological Society of Oxford, Volume 4, Issue 2, pp. 85-99.

HASHIM, I., 2005, Research report on independent child migration from northeastern to central Ghana, Research report, Development Research Centre on Migration, Globalization and Poverty, Brighton, University of Sussex.

-, 2006, The positives and negatives of children's independent migration: assessing the evidence and the debates, Working Paper T16, Development 
Research Centre on Migration, Globalization and Poverty, Brighton, University of Sussex.

-, 2007, Independent child migration and education in Ghana, Development and Change, 38(5), pp.911-931.

D Hashim, I. et D. THORSEN 201 Child Migration in Africa, London, Zedbooks, The Nordic Africa Institute.

HEAP, S., 1997, "Jaguda Boys": Pickpocketing in Ibadan, 1930-1960, Urban History, 24, pp.336-9.

HERTRICH, V. et M. LesClingand, 2007, Transition to adulthood and gender : changes in rural Mali, document de travail $n^{\circ} 140$, Paris, INED.

Hommes et Migrations, 2004, Enfants sans frontières, $n^{\circ} 1251$.

Hommes et Migrations, 2009, L'Afrique en mouvement, $\mathrm{n}^{\circ} 1279$.

Hommes et Migrations, 2010, Les migrations subsahariennes, $\mathrm{n}^{\circ}$ 1286-1287.

HONWANA, A. et DE BOECK, F., 2005, Makers and Breakers. Children and Youth in Postcolonial Africa, James Curey, Oxford.

en $\underbrace{}_{\text {Agents, in A. HONWANA et F. DE BOECK (éds.), Makers and Breakers. Children }}$ and Youth in Postcolonial Africa, James Curey, Oxford, pp.31-52.

HUERRE, P., 2001, L'histoire de l'adolescence : rôles et fonctions d'un artifice, Journal français de psychiatrie, 3(14), pp.6-8.

Hugon, Anne (éd.), 2004, Histoire des femmes en situation coloniale. Afrique et Asie, XXe siècle, Paris, Karthala.

Hunt, N. R., T. P. Liu, J. Quataert (éds.), 1997, Gendered Colonialism in African History, Oxford, Blackwell.

JAMES, A., 2007, Giving Voice to Children's Voices: Practices and Problems, Pitfalls and Potentials, American Anthropologist, vol. 109, n², pp. 261-272.

JAMES, A. et A. PROUT, (éds.), 1990, Constructing and Reconstructing Childhood: Contemporary Issues in the Sociological Study of Childhood, London and Washington DC, Falmer Press.

ILIFFE, J., 1987, The African Poor : A History, Cambridge, Cambridge University Press.

KANE, A., 2002, Senegal's Village Diaspora and the People Left Behind, in D. Bryceson et U. Vuorela (éds.), The Transnational Family: New European Frontiers and Global Networks, London, Berg Publishers, pp.245-263.

KLEIN, M. A. and R. RoBERTS, 1987, The Resurgence of Pawning in French West Africa during the Depression of the 1930s, African Economic History 16, pp. 23-37

KLEIN, M. A., 2011, Children and Slavery in the Western Sudan, in G. CAMPBELL, G., S. MIERS et J. MILLER (éds.), Child slaves in the modern world. Athens, Ohio University Press, pp.124-139.

KoM, D., 2009, Scolarisation, marriage et travail des enfants au Nord-Cameroun, Alternative Sud, vol 16, pp.117-146.

KUBIK, G., 1995, Kinderheit in aussereuropäischen Kulturen : Forschungsprobleme, -methoden und -ergebnisse, in E. Renner (éd.), Kinderwelten. Pädagogische, ethnologische und literaturwissenschaftliche Annäherungen, Weinheim, Deutscher Studien Verlag, pp.148-166. 
KUCZYNSKI, L. et E. RAZY, 2009, Anthropologie et migrations africaines en France : une généalogie des recherches, REMI, pp.79-100.

LALLEMAND, S., 1976, Génitrices et éducatrices mossi, L'Homme, 16(1), pp.109124.

-, 1977, Une famille Mossi, Paris-Ouagadougou, CNRS-CVRS, Recherches Voltaïques, 17.

-, 1988, Adoption, fosterage et alliance, Anthropologie et Sociétés, 12(2), pp.2540.

—, 1993, La circulation des enfants en société traditionnelle. Prêt, don échange, Paris, L'Harmattan.

-, 1994, Adoption et marriage. Les Kotokoli du Centre du Togo, Paris, L'Harmattan.

-, 1997, Enfances d'ailleurs, approche anthropologique, in M. GUIDETTI, S. Lallemand, M.-F. Morel, Enfances d'ailleurs, d'hier et d'aujourd'hui, Paris, Armand Colin, pp.7-57.

-, 2004, Mes mères et mes pères. Rapports entre filiation, terme de parenté et adoption-fosterage, in LEBLIC I. (éd.), De l'adoption : des pratiques de filiation différentes, Clermont-Ferrand, Presses universitaires Blaise Pascal, pp.299-321

LALLEMAND, S., et G. LE MOAL, 1981, Un petit sujet, Journal des Africanistes, 51 (1-2), pp.5-22.

LAMBERT, M.C., 2002, Longing for Exile. Migration and the Making of a Translocal Community in Senegal, West Africa, Portsmouth, Heinemann.

LANCY, D.F., 1996, Playing on the mother-ground. Cultural Routiness for Children's Development, New-York, Guilford Press.

-, 2008, The Anthropology of Childhood. Cherubs, Chattel, Changelings, New York, Cambridge University Press.

-, (à paraître), Unmasking Children's Agency, AnthropoChildren, 2.

LANGE, M.-F., 2001, Dynamiques scolaires contemporaines au Sud, in M.-F. Lange (éd.), Des écoles pour le Sud. Stratégies sociales, politiques étatiques et interventions du Nord, Autrepart, n 17, pp. 5-12.

LANGE, M.-F., 2003 (éd.), Enseignements, Cahiers d'Etudes Africaines, pp.169170.

LEBLIC, I. (éd.), 2004, De l'adoption: des pratiques de filiation différentes, Clermont-Ferrand, Presses universitaires Blaise Pascal.

LEE, C. J., 2009, Children in the Archives: Epistolary Evidence, Youth Agency, and the Social Meanings of "Coming of Age" in Interwar Nyasaland, Journal of Family History, 35, pp.25-49.

Le Jeune G., V. PiChÉ, J. PoIRIer, 2004, Towards a reconsideration of female migration patterns in Burkina Faso, Canadian Studies in Population, 31 (2), pp.145-177.

LORD, J., 2010, Spatial Approaches to the History of Child Labour in Colonial Ghana, Polyvocia - The SOAS Journal of Graduate Research, vol. 2., pp.31-45.

--, 2011, Child Labor in the Gold Coast : The Economics of Work, Education, and the Family in Late-Colonial African Childhoods, c. 1940-57, The Journal of the History of Childhood and Youth, vol. 4, $\mathrm{n}^{\circ} 1, \mathrm{pp} .88-115$.

MARFAING, L. et S. WIPPEL, éds., 2004, Les relations transsahariennes à l'époque contemporaine. Un espace en constante mutation, Paris, Karthala/ZMO. 
MASSART, G., 2009. Les pratiques de mobilité des enfants et des jeunes en Afrique de l'ouest. Analyse de leurs motivations et expériences, Working Paper, Symposium international Migration et mondialisation : 'Enjeux actuels et Défis futurs', Dakar, 2009. MBAYE, S. M., FALL A. S., 1996, Un tissu social qui se délite, un système qui se
et travail des enfants au Sénégal, in B. SCHLEMMER, (éd.), L'enfant exploité. Oppression. mise au travail. prolétarisation., Paris, Karthala, Orstom

$\mathrm{M}^{\circ}$ ClinTOCK, A., 1995, Imperial Leather: Race, Gender and Seuality in the Colonial Contest, New York, Routledge.

MAZzocchetTI, J., 2011, Fermeture des frontières et liens transnationaux : un terrain auprès de primo-migrants africains en Belgique, in $\mathrm{E}$. Razy et $\mathrm{V}$. BabyCollin (éds.), La famille transnationale dans tous ses états, Autrepart, n ${ }^{\circ} 57$, pp. 263-280.

MeILlassoux, C., 1986, Anthropologie de l'esclavage. Le ventre de fer et d'argent, Paris, Puf

Moch, L. P., 1987, Dividing Time: An Analytical Framework for Migration History Periodization, in Jan Lucassen et Leo Lucassen (éds.), Migration, Migration History. History, old Paradigms and New Perspectives, Berne, Peter Lang, pp.41-56.

MOITT, B., 2011, Slavery and Guardenship in Postemancipation Senegal. Colonial Legislation and Minors in Tutelle, 1848-1905, in G. CAMPBELL, G., S. MIERS et J. Miller (éds.), Child slaves in the modern world, Athens, Ohio University Press, pp.140-156.

MORAN, M. H., 1992, Civilized Servants : Child Fosterage and Training for Status among the Glebo of Liberia, in K. TRANBERG HANSEN éd., African Encounters with Domesticity, New Brunswick, Rutgers University Press, pp.98-115.

MONTGOMERY, H., 2008, An Introduction to Childhood: anthropological perspectives on children's lives, Oxford, Wiley-Blackwell.

NAGELS, C. et A. REA, A., 2007, Jeunes à perpète. Génération à problèmes ou problème de générations? , Louvain-La-Neuve, Academia Bruylant.

NDAO, M., 2003, La santé de l'enfant au Sénégal à l'époque coloniale. La variole et la rougeole de 1930 à 1960, Les cahiers histoire et civilisations, ${ }^{\circ} 1$, pp.85-98.

Ni Laoire, C., F. Carpena-MÉndez, N. Tyrrell et A. White, 2010, Introduction: Childhood and migration - mobilities, homes and belongings, Childhood, 17, n², pp.155-162.

Notermans, C., 2004, Sharing Home, Food, and Bed : Paths of Grandmotherhood in East Cameroon, Africa: Journal of the International African Institute, vol. $74, n^{\circ} 1$, Grandparents and Grandchildren, pp.6-27

-, 2008, The Emotional World of Kinship : Children's experiences of fosterage in East Cameroon, Childhood, 15, pp.355-377.

Oppong, C., G. AdABA, M. Bekombo-PRiso, J. Mogey (éds.), 1978, Marriage, Fertility and Parenthood in West Africa, Camberra, Australian National University.

Orellana, M.F., B. Thorne, A. CheE, et W.S.E. LAM, 2001, Transnational childhoods: the participation of children in processes of family migration, Social Problems, 48(4), pp.572-591. 
OYĚWuMI, O, 1997, The Invention of Women. Making an African Sense of Western Gender Discourses, Minneapolis, University of Minnesota Press.

Parsons, T., 2004, Race, Resistance and the Boy Scout Movement in British Colonial Africa, Athens, Ohio University Press

PEATRIK, A.-M., 1999, La vie à pas contés. Génération, âges et sociétés dans les hautes terres du Kénya, Nanterre, Société d'ethnologie.

Perry, D. L., 2004, Muslim Child Disciples, Global Civil Society, and Children's Rights in Senegal: The Discourses of Strategic Structuralism, Anthropological Quarterly, vol. 77, $\mathrm{n}^{\circ} 1$, pp.47-86

Pilon, M., Martin, J.-Y. et CARry, A., 2010, Introduction, in M. Pilon, J.-Y. MARTIN et A. CARRY, Le droit à l'éducation. Quelle universalité ?, Paris, Editions des Archives Contemporaines, pp.7-16.

PRICE, N., 1996, The Changing Value of Children among the Kikuyu of Central Province, Kenya, Africa 66, n³, pp.411-36

RABAIN, J., [1979] 1994, L'enfant du lignage. Du sevrage à la classe d'âge chez les Wolof au Sénégal, Paris, Payot.

RAZY, E., 2006, De quelques "retours soninké" aux différents âges de la vie. Circulations entre la France et le Mali, Journal des Anthropologues, n¹06-107, pp.337-354.

-, 2007a, Naître et devenir. Anthropologie de la petite enfance en pays soninké (Mali), Nanterre, Société d'Ethnologie.

-, 2007b, Les "sens contraires" de la migration. La circulation des jeunes filles d'origine soninké entre la France et le Mali, Journal des Africanistes, 77(2), pp.19-43.

—, 2007c, Les "réfugiées de l'intérieur". Excision et mariage précoce contraint entre la France et le Mali, in J. Freedman et J. VALluY (éds.), Persécutions des femmes. Savoirs, mobilisations, protections, Paris, Editions Du Croquant (Collection TERRA), pp.189-204.

- 2010, La famille dispersée: une configuration pluriparentale oubliée ?, L'Autre, 11(3), pp.331-339.

-, (sous presse), La pratique des sentiments. Des corps dans les constellations de la petite enfance (Pays soninké, Mali), in D. BONNET, C. Rollet, et C.-E. DE SUREMAIN (éds), Modèles d'enfances. Successions, transformations, croisements, Paris, Editions des Archives contemporaines.

RAZY, E. et V. BABY-COLlIN, V., 2011, Introduction, in E. RAZY et V. BABYCOLLIN (éds.), La famille transnationale dans tous ses états, Autrepart, n ${ }^{\circ} 57$, pp.7-22.

RICH, J., 2010, Searching for Success : Boys, Family Aspirations, and Opportunities in Gabon, ca. 1900-1940, Journal of Family History, 35/1, pp.7-24.

RIESMAN, P., 1992, First find your child a good mother. The construction of self in two african communities, New Brunswick New Jersey, Rutgers University Press.

RiviĖRE, C., 1990 Union et procréation en Afrique. Rites de la vie chez les Evé du Togo, Paris, L'Harmattan/Connaissance des hommes.

RODET, M., 2006, C'est le regard qui fait l'histoire. Comment utiliser des archives coloniales qui nous renseignent malgré elles sur l'histoire des femmes africaines, Terrains et Travaux, $\mathrm{n}^{\circ} 10$, pp.18-34. 
—, 2009, Les migrantes ignorées du Haut-Sénégal (1900-1946), Paris, Karthala.

-, 2011, Genre, Islam et pluralisme juridique au Soudan français (1900-1925), Outre-Mers, T.99, no370-371, pp.173-183.

Rout Biel, M. 2004, African Kids. Between Warlords, Child Soldiers and Living on the Street, Frankfurt, Peter Lang.

Rogers, B., 1980, The Domestication of Women, Discrimination in Developing Societies, London, Kogan Page.

ROLlET, C., 2001, Les enfants au XIXè siècle, Paris, Hachette.

Rollet-ECHALIER, C., 1990, La politique à l'égard de la petite enfance sous la troisième république, Paris, INED/Presses Universitaires de France.

× SAYAD A. \$1977\%, Les trois âges de l'émigration algérienne en France, Actes de la recherche en Sciences Sociales, $\mathrm{n}^{\circ} 15,59,82$.

f SChePer-Hughes, N. et C. SARGent, C. ('eds), 1998, Small wars: The Cultural Politics of Childhood, Berkeley, University of California Press.

SCHLEMmer, B. (éd.), 1996, L'enfant exploité. Oppression, mise au travail, prolétarisation. Paris, Karthala, Orstom.

SCHMIDT, E., 1992, Peasants, Traders, and Wives : Shona Women in the History of Zimbabwe, 1870-1939. Portsmouth, Heinemann.

SCHMITZ, J., 2008, Migrants oues-africains vers l'Europe : historicité et espace moral $\%$ in J. Schmitz (éd.), Migrants ouest-africains : miséreux, aventuriers ou notables ?, Politique africaine, n' 9 , pp.5-15.

SEGALEN, M., 2010, A qui appartiennent les enfants?, Paris, Tallandier

SHADLE, B. L., 2006, "Girl Cases" : Marriage and Colonialism in Gusiiland, Kenya, 1890-1970, Portsmouth, Heinemann.

Sow, A., 2009, Les de la politique educative coloniak De l'école assimilationniste à l'école populaire au Sénégal : 1817-1946, Historiens et Géographes du Sénégal, n ${ }^{\circ}$, L'esclavage et ses traites en Afrique, discours méncivels travirs interdits, pp.58-68.

StePHens, S. (éd.), 1995, Children and the Politics of Culture, Princeton, Princeton - Veliquniversity Press.

non 2 SUREMAIN (DE), C.-E., 2007, L'entourage nourricier de l'enfant. À partir virgule d'exemples en Bolivie et au Congo, L'Autre, 8(3), pp.349-366.

TAMBA, M., 2003, Le travail des enfants dans l'artisanat de production à Dakar. Les apprentis cordonniers de la Médina, Les cahiers histoire et civilisations, $\mathrm{n}^{\circ} 1$, pp.99-104.

Terretta, M., 2007, A Miscarriage of Revolution: Cameroonian Women and Nationalism, in O. GOERG, M. RODET, N. VINCE (éds.), Fracturing Binarisms : Gender and Colonialisms in Africa, Stichproben, $\mathrm{n}^{\circ} 12$

THIERCE, A., 1999, Histoire de l'adolescence (1850-1914), Paris, Belin.

THIOUB, I., 2003, La gestion de la marginalité juvénile dans la colonie du Sénégal, Les cahiers histoire et civilisations, ${ }^{\circ} 1, \mathrm{pp} .117-129$.

THorsen, D., 2006, Child migrants in transit. Strategies to become adult in rural Burkina Faso, in C. Christiansen, M. UTAS et H. E. Vigh (éds.), Navigating Youth, Generating Adulthood: Social Becoming in an African Context, Uppsala, Nordic africa Institute, pp.88-114.

_-, 2007, "If Only I Get Enough Money for a Bicycle!" A Study of Childhoods, Migration and Adolescent Aspirations Against a Backdrop of Exploitation and 
Trafficking in Burkina Faso, Working Paper T21, Brighton, Development Research Centre on Migration, Globalisation and Poverty.

TIMERA, M., 2001, Les migrations des jeunes en Afrique noire : Affirmation de soi et émancipation, in Les jeunes, hantise de l'espace public dans les sociétés du Sud ?, Autrepart, n ${ }^{\circ} 8$, pp.37-59.

UnGRUHE, C., 2010, Symbols of success: Youth, peer pressure and the role of adulthood among juvenile male return migrants in Ghana, Childhood, $\mathrm{n}^{\circ} 17$, pp.259-271.

VIDAL, L., 2000, Femmes en temps de sida. Expériences d'Afrique, Presses universitaires de France, Paris.

Waller, 2006, Rebellious Youth in Colonial Africa, Journal of African History, 47, pp.77-92.

WEISS, F., 1993, Von der Schwierigkeit über Kinder zu forschen. Die latmul in Papua-Neuguinea, in M.-J. VAN DE LOO et M. REINHART, Kinder. Ethnologische Forschungen in fiinf Kontinenten, München, Trickster Verlag, , pp.96-155.

-, 1995, Kinder erhalten das Wort. Aussagen von Kindern in Ethnologie, in E. RENNER (éd.), Kinderwelten. Pädagogische, ethnologische und literaturwissenschaftliche Annäherungen, Weinheim, Deutscher Studien Verlag, pp.133-147.

WHITE, O., 2004 [1999], Children of the French Empire: Miscegenation and Colonial Society in French West Africa, 1895-1960, Oxford, Oxford University Press.

WHITEHEAD, A. et I. HASHIM, 2005, Children and migration : background paper for DflD migration team, London, Department for International Development.

WHITEHEAD, A, I. HASHIM et I. IVERSEN, 2007, Child migration, child agency and inter-generational relations in Africa and South Asia, Working Paper T24, Development Research Centre on Migration, Globalization and Poverty, Brighton, University of Sussex.

WhitehousE, B., 2009, Transnational childrearing and the preservation of transnational identity in Brazzaville, Congo, Global Networks, 9(1), pp.82-99.

WoOdward, W., P. Hayes, G. Minkley (éds.), 2002, Deep hiStories : Gender and Colonialism in Southern Africa, Amsterdam, Rodopi.

WouAngo, J., 2011, Travail des enfants et droit à l'éducation au Burkina Faso L'exemple de la carrière de Pissy, in Les enfants hors l'école, Cahiers de la recherche sur l'éducation et les savoirs, 10, pp. 127-141.

(sous presse), L'éternel retour du travail des enfants dans les politiques publiques au Burkina Faso : perspective historique, in D. BONNET, C. RolleT, EMAIN (de) C.-E. (éds.), Modèles d'enfances. Successions, transformations, roisements, Paris, Editions des Archives contemporaines. 